\begin{tabular}{|c|l|}
\hline Title & Convergence and optimal control problems of nonlinear evolution equations governed by time dependent operator \\
\hline Author(s) & Yamazaki, Noriaki \\
\hline Citation & Hokkaido University Preprint Series in Mathematics, 894, 1-27 \\
\hline Issue Date & 2008 \\
\hline DOI & 10.14943/84044 \\
\hline Doc URL & http:/hdl.handle.net/2115/69703 \\
\hline Type & bulletin (article) \\
\hline File Information & pre894.pdf \\
\hline
\end{tabular}

Instructions for use 


\title{
Convergence and optimal control problems of nonlinear evolution equations governed by time-dependent operator
}

\author{
NORIAKI YAMAZAKI \\ Department of Mathematical Science, Common Subject Division \\ Muroran Institute of Technology, Muroran, Japan \\ E-mail: noriaki@mmm.muroran-it.ac.jp
}

\begin{abstract}
We study an abstract nonlinear evolution equation governed by time-dependent operator of subdifferential type in a real Hilbert space. In this paper we discuss the convergence of solutions to our evolution equations. Also, we investigate the optimal control problems of nonlinear evolution equations. Moreover, we apply our abstract results to a quasilinear parabolic PDE with a mixed boundary condition.
\end{abstract}

\section{Introduction}

We study an abstract nonlinear evolution equation in a real Hilbert space $H$ of the form:

$$
u^{\prime}(t)+\partial \varphi^{t}(u(t) ; u(t))+G(t, u(t)) \ni f(t) \quad \text { in } H, \text { a.e. } t \in(0, T),
$$

where $0<T<+\infty, u^{\prime}(t):=\frac{d}{d t} u(t), f$ is a given $H$-valued function, and $G(t, \cdot)$ is a single-valued perturbation small relative to $\varphi^{t}(\cdot ; \cdot)$.

Here, for each $t \in[0, T]$, a function $\varphi^{t}(\cdot ; \cdot): H \times H \rightarrow \mathbb{R} \cup\{\infty\}$ is given such that for all $w \in H, \varphi^{t}(w ; \cdot): H \rightarrow \mathbb{R} \cup\{\infty\}$ is a proper, l.s.c. (lower semi-continuous) and convex function, and $\partial \varphi^{t}(w ; \cdot)$ is its subdifferential operator in $H$, i.e., $z^{*} \in \partial \varphi^{t}(w ; z)$ if and only if

$$
z \in D\left(\varphi^{t}(w ; \cdot)\right) \text { and }\left(z^{*}, y-z\right) \leq \varphi^{t}(w ; y)-\varphi^{t}(w ; z) \text { for all } y \in H .
$$

For a proper, l.s.c. and convex function $\psi^{t}(\cdot): H \rightarrow \mathbb{R} \cup\{\infty\}$, many mathematicians studied the nonlinear evolution equation of the form:

$$
u^{\prime}(t)+\partial \psi^{t}(u(t)) \ni f(t) \quad \text { in } H \text {, a.e. } t \in(0, T) .
$$

For various aspects of (1.2), we refer to [1, 3, 7, 9, 10, 13, 15, 23, 27, 30]. For instance, Kenmochi [13] showed the existence-uniqueness, the stability and the convergence of solutions to (1.2). Also, Hu-Papageorgiou [10] studied the optimal control problems to $(1.2)$.

Key words and phrases. Nonlinear evolution equation; Convergence; Optimal control; Subdifferential; Quasilinear parabolic PDE media. 
For the nonmonotone perturbation $G(t, \cdot)$, Otani [21] has already shown the existence of solution to the equation of the form:

$$
u^{\prime}(t)+\partial \psi^{t}(u(t))+G(t, u(t)) \ni f(t) \quad \text { in } H \text {, a.e. } t \in(0, T) .
$$

The large-time behavior of solutions for (1.3) was discussed by [25] from the view-point of attractors. For another works of (1.3), we refer to [14, 22, 25, 28], for instance.

Note that the time-dependent function $\varphi^{t}(z ; z)$ in (1.1) is not convex in $z$. So, we can not apply the abstract theory established by Ôtani [21] to our equation (1.1). Recently, the author [29] proved the existence and uniqueness of solutions to (1.1). For similar works, we refer to $[11,26]$ for instance.

The first main object of this paper is to establish the convergence result of solutions to (1.1). More precisely, we consider the following Cauchy problems, denoted by $(\mathrm{CP})$ and $(\mathrm{CP})_{n}(n \in \mathbb{N})$ :

$$
(\mathrm{CP})\left\{\begin{array}{l}
u^{\prime}(t)+\partial \varphi^{t}(u(t) ; u(t))+G(t, u(t)) \ni f(t) \quad \text { in } H, \text { a.e. } t \in(0, T), \\
u(0)=u_{0},
\end{array}\right.
$$

and

$$
(\mathrm{CP})_{n}\left\{\begin{array}{l}
u_{n}^{\prime}(t)+\partial \varphi_{n}^{t}\left(u_{n}(t) ; u_{n}(t)\right)+G_{n}\left(t, u_{n}(t)\right) \ni f_{n}(t) \quad \text { in } H, \text { a.e. } t \in(0, T), \\
u_{n}(0)=u_{0, n},
\end{array}\right.
$$

where $u_{0} \in H$ is the given initial condition. Also, for each $n \in \mathbb{N}, \varphi_{n}^{t}(\cdot ; \cdot)$ is a timedependent function introduced as above, $G_{n}(t, \cdot)$ is a single-valued perturbations small relative to $\varphi_{n}^{t}(\cdot ; \cdot)$, the function $f_{n} \in L^{2}(0, T ; H)$ is given, and $u_{0, n} \in H$ is the given initial condition. Then, assuming that $\varphi_{n}^{t}(\cdot ; \cdot), G_{n}(t, \cdot), f_{n}(t)$ and $u_{0, n}$ converge to $\varphi^{t}(\cdot ; \cdot), G(t, \cdot)$, $f(t)$ and $u_{0}$, respectively, (in some senses) as $n \rightarrow \infty$, we shall show that the solution $u_{n}$ of $(\mathrm{CP})_{n}$ converges to one $u$ of $(\mathrm{CP})$ in an appropriate sense.

The second object of this paper is to consider the optimal control problem to (CP), denoted by (OP), as follows:

Problem (OP). Find a function (optimal control) $f^{*} \in L^{2}(0, T ; H)$ such that

$$
J\left(f^{*}\right)=\inf _{f \in L^{2}(0, T ; H)} J(f) .
$$

Here, $J(\cdot)$ is a cost functional defined by:

$$
J(f):=\frac{1}{2} \int_{0}^{T}\left|\left(u-u_{d}\right)(t)\right|_{H}^{2} d t+\frac{1}{2} \int_{0}^{T}|f(t)|_{H}^{2} d t, \quad \forall f \in L^{2}(0, T ; H),
$$

where $|\cdot|_{H}$ denotes the norm of $H$, the main parameter $f$ is called the control, $u_{d}$ is a given desired target profile in $L^{2}(0, T ; H)$, and $u$ is a unique solution to $(\mathrm{CP})$ with the control $f \in L^{2}(0, T ; H)$.

Furthermore, for each $n \in \mathbb{N}$ we study the optimal control problem to $(\mathrm{CP})_{n}$, denoted by $(\mathrm{OP})_{1, n}$, as follows:

Problem $(\mathrm{OP})_{1, n}$. Find a function (optimal control) $f_{1, n}^{*} \in L^{2}(0, T ; H)$ such that

$$
J_{1, n}\left(f_{1, n}^{*}\right)=\inf _{f \in L^{2}(0, T ; H)} J_{1, n}(f) .
$$


Here, $J_{1, n}(\cdot)$ is a cost functional defined by:

$$
J_{1, n}(f):=\frac{1}{2} \int_{0}^{T}\left|\left(u_{n}-u_{d}\right)(t)\right|_{H}^{2} d t+\frac{1}{2} \int_{0}^{T}|f(t)|_{H}^{2} d t, \quad \forall f \in L^{2}(0, T ; H),
$$

where $u_{n}$ is a unique solution to $(\mathrm{CP})_{n}$ with the control $f \in L^{2}(0, T ; H)$.

Also, for each $n \in \mathbb{N}$ we study the another type of optimal control problem to $(\mathrm{CP})_{n}$, denoted by $(\mathrm{OP})_{2, n}$, as follows:

Problem $(\mathrm{OP})_{2, n}$. Find a function (optimal control) $f_{2, n}^{*} \in L^{2}(0, T ; H)$ such that

$$
J_{2, n}\left(f_{2, n}^{*}\right)=\inf _{f \in L^{2}(0, T ; H)} J_{2, n}(f) .
$$

Here, $J_{2, n}(\cdot)$ is a cost functional defined by:

$$
\begin{array}{r}
J_{2, n}(f):=\frac{1}{2} \int_{0}^{T}\left|\left(u_{n}-u_{d}\right)(t)\right|_{H}^{2} d t+\frac{1}{2} \int_{0}^{T}|f(t)|_{H}^{2} d t+\frac{1}{2} \int_{0}^{T}\left|\left(f-f^{*}\right)(t)\right|_{H}^{2} d t \\
\text { for any } f \in L^{2}(0, T ; H),
\end{array}
$$

where $f^{*}$ is the optimal control of $(\mathrm{OP})$, and $u_{n}$ is a unique solution to $(\mathrm{CP})_{n}$ with the control $f \in L^{2}(0, T ; H)$.

Then, the final aim of this present paper is to consider the relationship between (OP) and $(\mathrm{OP})_{1, n}$. Also, we study the relationship between $(\mathrm{OP})$ and $(\mathrm{OP})_{2, n}$.

So, the main novelties found in this paper are:

(i) to prove that the unique solution of $(\mathrm{CP})_{n}$ converges to one of $(\mathrm{CP})$ in some appropriate sense;

(ii) to prove the existence of optimal controls for (OP), (OP $)_{1, n}$ and $(\mathrm{OP})_{2, n}$;

(iii) to show the relationship between $(\mathrm{OP})$ and $(\mathrm{OP})_{1, n}$;

(iv) to show the relationship between $(\mathrm{OP})$ and $(\mathrm{OP})_{2, n}$.

The plan of this paper is as follows. In the next Section 2, we present the results on the existence and uniqueness of solutions to (CP). In Section 3, we state the main results in this paper. In Section 4, we prove the convergence result (Theorem 3.2) of solutions, which corresponds to the item (i) listed in the above. In Section 5, we prove the main result (Theorem 3.3) on the existence of the optimal control to (OP). Also, we show Theorem 3.4 (resp. Theorem 3.5), which is concerned with the relationship between $(\mathrm{OP})$ and $(\mathrm{OP})_{1, n}$ (resp. (OP) and $(\mathrm{OP})_{2, n}$ ). In the final Section 6, we apply our abstract results to a quasilinear parabolic PDE with a mixed boundary condition.

\section{Notation}

Throughout this paper, let $H$ be a real Hilbert space with norm $|\cdot|_{H}$ and inner product $(\cdot, \cdot)$. For a proper, l.s.c. and convex function $\psi$ on $H$, we use the notations $D(\psi), \partial \psi$ and $D(\partial \psi)$ to indicate the effective domain, subdifferential and its domain of $\partial \psi$, respectively. For their precise definitions and basic properties, see a monograph by Brézis [6]. 


\section{Assumptions and solvability results}

In this section, we consider the Cauchy problem (CP) for (1.1) of the following form:

$$
(\mathrm{CP})\left\{\begin{array}{l}
u^{\prime}(t)+\partial \varphi^{t}(u(t) ; u(t))+G(t, u(t)) \ni f(t) \quad \text { in } H, \text { a.e. } t \in(0, T), \\
u(0)=u_{0},
\end{array}\right.
$$

where $T$ is a given positive number, a function $\varphi^{t}(\cdot ; \cdot)$ is introduced in Section $1, G(t, \cdot)$ is a single-valued perturbation small relative to $\varphi^{t}(\cdot ; \cdot), f \in L^{2}(0, T ; H)$ is the given function, and $u_{0} \in H$ is the given initial condition.

We begin by defining the solutions of $(\mathrm{CP})$. In the rest, we denote by $\left(\mathrm{CP} ; f, u_{0}\right)$ our problem $(\mathrm{CP})$ when the data of the forcing term $f$ and the initial condition $u_{0}$ are specified:

Definition 2.1. Given $f \in L^{2}(0, T ; H)$ and $u_{0} \in H$, the function $u:[0, T] \rightarrow H$ will be called a solution to $\left(\mathrm{CP} ; f, u_{0}\right)$, if $u \in W^{1,2}(0, T ; H), u(0)=u_{0}, u(t) \in D\left(\partial \varphi^{t}(u(t) ; \cdot)\right.$ and $f(t)-u^{\prime}(t)-G(t, u(t)) \in \partial \varphi^{t}(u(t) ; u(t))$ for a.e. $t \in[0, T]$, namely

$$
\begin{gathered}
\left(f(t)-u^{\prime}(t)-G(t, u(t)), y-u(t)\right) \leq \varphi^{t}(u(t) ; y)-\varphi^{t}(u(t) ; u(t)) \\
\text { for any } y \in H \text { and a.e. } t \in[0, T] .
\end{gathered}
$$

Now, we give the assumptions on $\varphi^{t}(\cdot ; \cdot)$. To do so, let $\left\{\alpha_{r}\right\}:=\left\{\alpha_{r} ; r>0\right\}$ be a family of functions $\alpha_{r} \in W^{1,2}(0, T)$ with parameter $r>0$. Then, with this family $\left\{\alpha_{r}\right\}$, we specify a class $\Phi\left(\left\{\alpha_{r}\right\}\right)$ of all families $\left\{\varphi^{t}\right\}:=\left\{\varphi^{t} ; t \in[0, T]\right\}$ of time-dependent functions $\varphi^{t}(\cdot ; \cdot)$ on $H \times H$ as follows.

Definition 2.2. We denote by $\left\{\varphi^{t}\right\} \in \Phi\left(\left\{\alpha_{r}\right\}\right)$ the set of all time-dependent functions $\varphi^{t}(\cdot ; \cdot)$ from $H \times H$ into $\mathbb{R} \cup\{\infty\}$ satisfying the following conditions:

$(\Phi 1)$ For each $w \in H$ and $t \in[0, T], \varphi^{t}(w ; \cdot): H \rightarrow \mathbb{R} \cup\{\infty\}$ is a proper, 1.s.c. and convex function;

(Ф2) There exists a positive constant $C_{1}>0$ such that

$$
\varphi^{t}(w ; z) \geq C_{1}|z|_{H}^{2}, \quad \forall t \in[0, T], \forall w \in H, \forall z \in D\left(\varphi^{t}(w ; \cdot)\right) ;
$$

(Ф3) For each $t \in[0, T], w \in H$ and $k>0$, the level set $\left\{z \in H ; \varphi^{t}(w ; z) \leq k\right\}$ is compact in $H$;

(Ф4) $D\left(\varphi^{t}(w ; \cdot)\right)$ is independent of $w \in H$ for any $t \in[0, T]$;

(Ф5) For each $w \in H, r>0, s, t \in[0, T]$ with $s \leq t$ and $z \in D\left(\varphi^{s}(w ; \cdot)\right)$ with $|z|_{H} \leq r$, there exists an element $\tilde{z} \in D\left(\varphi^{t}(w ; \cdot)\right)$ such that

$$
|\tilde{z}-z|_{H} \leq\left|\alpha_{r}(t)-\alpha_{r}(s)\right|\left(1+\varphi^{s}(w ; z)^{\frac{1}{2}}\right)
$$

and

$$
\varphi^{t}(w ; \tilde{z})-\varphi^{s}(w ; z) \leq\left|\alpha_{r}(t)-\alpha_{r}(s)\right|\left(1+\varphi^{s}(w ; z)\right)
$$


(Ф6) There is a positive constant $C_{2}>0$ such that

$$
\begin{gathered}
\left|\varphi^{t}\left(w_{1} ; z\right)-\varphi^{t}\left(w_{2} ; z\right)\right| \leq C_{2}\left|w_{1}-w_{2}\right|_{H}\left(\varphi^{t}(0 ; z)^{\frac{1}{2}}+\left|w_{1}\right|_{H}+\left|w_{2}\right|_{H}+1\right), \\
\forall t \in[0, T], \forall w_{i} \in H(i=1,2), \forall z \in D\left(\varphi^{t}(0, \cdot)\right) .
\end{gathered}
$$

Remark 2.3. For each $w \in H$, the assumption $(\Phi 5)$ implies the standard time-dependence condition of convex functions (cf. [3, 13, 27, 30]).

Next, we introduce the class $\mathcal{G}\left(\left\{\varphi^{t}\right\}\right)$ of time-dependent perturbation $G(t, \cdot)$ associated with $\left\{\varphi^{t}\right\} \in \Phi\left(\left\{a_{r}\right\}\right)$.

Definition 2.4. $\{G(t, \cdot)\} \in \mathcal{G}\left(\left\{\varphi^{t}\right\}\right)$ if and only if $G(t, \cdot)$ is a single-valued operator from $D(G(t, \cdot)) \subset H$ into $H$ which fulfills the following conditions:

(G1) $D\left(\varphi^{t}(0 ; \cdot)\right) \subset D(G(t, \cdot)) \subset H$ for all $t \in[0, T]$, and $G(\cdot, v(\cdot))$ is strongly measurable on $[0, T]$ for any $v \in L^{2}(0, T ; H)$ with $v(t) \in D\left(\varphi^{t}(0 ; \cdot)\right)$ for a.e. $t \in[0, T]$;

(G2) There are positive constants $C_{3}>0$ and $C_{4}>0$ such that

$$
|G(t, z)|_{H}^{2} \leq C_{3} \varphi^{t}(z ; z)+C_{4}, \quad \forall t \in[0, T], \forall z \in D\left(\varphi^{t}(0 ; \cdot)\right)
$$

(G3) (Demi-closedness) If $\left\{t_{n}\right\} \subset[0, T],\left\{z_{n}\right\} \subset H, t_{n} \rightarrow t, z_{n} \rightarrow z$ in $H$ (as $n \rightarrow \infty$ ) and $\left\{\varphi^{t_{n}}\left(z_{n}, z_{n}\right)\right\}$ is bounded, then $G\left(t_{n}, z_{n}\right) \rightarrow G(t, z)$ weakly in $H$ as $n \rightarrow \infty$.

By the slight modification of [29, Theorem 1], we can show the existence of solutions to $(\mathrm{CP})$ as follows:

Proposition 2.5 (cf. [29, Theorem 1]). Let T be any positive number. Assume $\left\{\varphi^{t}\right\} \in$ $\Phi\left(\left\{\alpha_{r}\right\}\right)$ and $\{G(t, \cdot)\} \in \mathcal{G}\left(\left\{\varphi^{t}\right\}\right)$. Then, for each $f \in L^{2}(0, T ; H)$ and $u_{0} \in D\left(\varphi^{0}(0 ; \cdot)\right)$, the Cauchy problem $\left(\mathrm{CP} ; f, u_{0}\right)$ has at least one solution $u$ on $[0, T]$. Moreover, there are positive constants $N_{1}=N_{1}(T)$ and $N_{2}=N_{2}(T)$, dependent on $T$, such that

$$
\sup _{t \in[0, T]}|u(t)|_{H} \leq N_{1}\left(\left|u_{0}\right|_{H}+|f|_{L^{2}(0, T ; H)}+1\right)
$$

and

$$
\sup _{t \in[0, T]} \varphi^{t}(u(t) ; u(t))+\int_{0}^{T}\left|u^{\prime}(t)\right|_{H}^{2} d t \leq N_{2}\left(\varphi^{0}\left(u_{0} ; u_{0}\right)+|f|_{L^{2}(0, T ; H)}^{2}+1\right) .
$$

Note that the assumptions of $\varphi^{t}(\cdot, \cdot)$ and $G(t, \cdot)$ are slight different from ones in [29]. But, by the similar technique in [16, 17, 29], namely, by using the fixed point arguments, we can show Proposition 2.5.

In fact, for a given positive number $T>0$, we put a Banach space

$$
E(T) \equiv\left\{w \in W^{1,2}(0, T ; H) ; \sup _{t \in[0, T]} \varphi^{t}(0 ; w(t))<+\infty\right\} .
$$


By Remark 2.3 and the abstract theory established by Kenmochi [13], we can get a unique solution $h \in W^{1,2}(0, T ; H)$ to the following Cauchy problem:

$$
\left\{\begin{array}{l}
h^{\prime}(t)+\partial \varphi^{t}(0 ; h(t)) \ni f(t) \quad \text { in } H, \text { a.e. } t \in(0, T) \\
h(0)=u_{0}
\end{array}\right.
$$

Then, we easily observe from [13, Theorem 1.1.2] that $\sup _{t \in[0, T]} \varphi^{t}(0 ; h(t))<+\infty$, hence, we observe that $E(T) \ni h$, namely, $E(T) \neq \emptyset$.

Here, for each $w \in E(T)$ let us consider the following Cauchy problem, denoted by $\left(\mathrm{CP} ; f, u_{0}\right)_{w}$ :

$$
\left(\mathrm{CP} ; f, u_{0}\right)_{w}\left\{\begin{array}{l}
u^{\prime}(t)+\partial \varphi^{t}(w(t) ; u(t)) \ni f(t)-G(t, w(t)) \text { in } H, \text { a.e. } t \in(0, T), \\
u(0)=u_{0} .
\end{array}\right.
$$

To show the existence-uniqueness of solution to $\left(\mathrm{CP} ; f, u_{0}\right)_{w}$, we give the key lemma.

Lemma 2.6 (cf. [29, Lemma 1]). Suppose all the same conditions in Proposition 2.5. For each $w \in E(T)$, we take a positive constant $R>0$ such that $\sup _{t \in[0, T]}|w(t)|_{H} \leq R$, and put

$$
\psi_{w}^{t}(z):=\varphi^{t}(w(t) ; z) \quad \text { for } z \in H .
$$

Then, there is a positive constant $N_{3}>0$, independent of $w$, satisfying the following:

$(\star)$ For each $r>0, s, t \in[0, T]$ with $s \leq t$ and $z \in D\left(\psi_{w}^{s}\right)$ with $|z|_{H} \leq r$, there exists $\tilde{z} \in D\left(\psi_{w}^{t}\right)$ such that

$$
|\tilde{z}-z|_{H} \leq\left|\alpha_{r}(t)-\alpha_{r}(s)\right|\left(1+\psi_{w}^{s}(z)^{\frac{1}{2}}\right)
$$

and

$$
\begin{aligned}
& \psi_{w}^{t}(\tilde{z})-\psi_{w}^{s}(z) \\
\leq & N_{3}(1+R)\left\{\left|\alpha_{r}(t)-\alpha_{r}(s)\right|\left(1+\psi_{w}^{s}(z)\right)+|w(t)-w(s)|_{H}\left(1+\psi_{w}^{s}(z)\right)^{\frac{1}{2}}\right\} .
\end{aligned}
$$

Proof. By the similar argument in [29, Lemma 1], we can show Lemma 2.6. In fact, we take $w=w(s)$ in $(\Phi 5)$. Then, for each $r>0, s, t \in[0, T]$ with $s \leq t$ and $z \in D\left(\varphi^{s}(w(s) ; \cdot)\right.$ with $|z|_{H} \leq r$, there exists $\tilde{z} \in D\left(\varphi^{t}(w(s) ; \cdot)\right)$ such that

$$
|\tilde{z}-z|_{H} \leq\left|\alpha_{r}(t)-\alpha_{r}(s)\right|\left(1+\varphi^{s}(w(s) ; z)^{\frac{1}{2}}\right)
$$

and

$$
\varphi^{t}(w(s) ; \tilde{z})-\varphi^{s}(w(s) ; z) \leq\left|\alpha_{r}(t)-\alpha_{r}(s)\right|\left(1+\varphi^{s}(w(s) ; z)\right) .
$$

Here, note from $(\Phi 4)$ that

$$
z \in D\left(\varphi^{s}(w(s) ; \cdot)=D\left(\psi_{w}^{s}\right), \quad \tilde{z} \in D\left(\varphi^{t}(w(s) ; \cdot)\right)=D\left(\psi_{w}^{t}\right) .\right.
$$


Also, from $(\Phi 6)$ and $w \in E(T)$ with $\sup _{t \in[0, T]}|w(t)|_{H} \leq R$, we note that

$$
\begin{aligned}
\varphi^{t}(0 ; \tilde{z}) & \leq 2 \varphi^{t}(w(t) ; \tilde{z})+C_{2}^{2}|w(t)|_{H}^{2}+2 C_{2}|w(t)|_{H}^{2}+2 C_{2}|w(t)|_{H} \\
& \leq 2 \psi_{w}^{t}(\tilde{z})+C_{2}^{2} R^{2}+2 C_{2} R^{2}+2 C_{2} R .
\end{aligned}
$$

Then, we observe from $(2.5),(2.7)$ and $(\Phi 6)$ that there is a positive number $N_{4}>0$ independent of $w$ satisfying the following:

$$
\begin{aligned}
& \psi_{w}^{t}(\tilde{z})-\psi_{w}^{s}(z) \\
=\quad & \varphi^{t}(w(t) ; \tilde{z})-\varphi^{t}(w(s) ; \tilde{z})+\varphi^{t}(w(s) ; \tilde{z})-\varphi^{s}(w(s) ; z) \\
\leq \quad & N_{4}\left\{|w(t)-w(s)|_{H} \psi_{w}^{t}(\tilde{z})^{\frac{1}{2}}+\right. \\
& (1+R)|w(t)-w(s)|_{H} \\
& \left.+\left|\alpha_{r}(t)-\alpha_{r}(s)\right|\left(1+\psi_{w}^{s}(z)\right)\right\} .
\end{aligned}
$$

Here, let us give the estimate the term $\psi_{w}^{t}(\tilde{z})^{\frac{1}{2}}$ in the right hand side of (2.8). From (2.8) and $w \in E(T)$ with $\sup _{t \in[0, T]}|w(t)|_{H} \leq R$, it follows that

$$
\psi_{w}^{t}(\tilde{z}) \leq N_{5}\left\{(1+R)^{2}\left(1+\psi_{w}^{s}(z)\right)+\left|\alpha_{r}(t)-\alpha_{r}(s)\right|\left(1+\psi_{w}^{s}(z)\right)\right\}
$$

for some constant $N_{5}>0$ independent of $w$. Therefore, using (2.9) in the right hand side of (2.8), and by (2.4)-(2.6), we get this Lemma 2.6 for some constant $N_{3}>0$ independent of $w$.

Lemma 2.7. Suppose all the same conditions in Proposition 2.5. Then, for each $w \in$ $E(T)$, the Cauchy problem $\left(\mathrm{CP} ; f, u_{0}\right)_{w}$ has a unique solution $u$ on $[0, T]$.

Proof. Note that $\left(\mathrm{CP} ; f, u_{0}\right)_{w}$ can be regarded as the Cauchy problem for the nonlinear evolution equation of the form:

$$
\left\{\begin{array}{l}
u^{\prime}(t)+\partial \psi_{w}^{t}(u(t)) \ni f(t)-G(t, w(t)) \quad \text { in } H, \text { a.e. } t \in(0, T), \\
u(0)=u_{0} .
\end{array}\right.
$$

We observe from $(\Phi 6)$ and $(\mathrm{G} 2)$ that for any $w \in E(T)$ with $\sup _{t \in[0, T]}|w(t)|_{H} \leq R$,

$$
\begin{aligned}
& \int_{0}^{T}|G(t, w(t))|_{H}^{2} d t \\
\leq & \int_{0}^{T}\left\{C_{3} \varphi^{t}(w(t) ; w(t))+C_{4}\right\} d t \\
\leq & T C_{3}\left\{2 \sup _{t \in[0, T]} \varphi^{t}(0 ; w(t))+\frac{C_{2}^{2} R^{2}}{4}+C_{2} R^{2}+C_{2} R\right\}+T C_{4} \\
< & +\infty,
\end{aligned}
$$

which implies that $f-G(\cdot, w(\cdot)) \in L^{2}(0, T ; H)$. Moreover, we see from Lemma 2.6 that the time-dependence condition of $\psi_{w}^{t}$ holds. Therefore, by taking account of the assumption 
$(\Phi 1)$, we can apply the abstract theory established by Kenmochi [13]. Thus, we get the existence-uniqueness of solution $u$ for $\left(\mathrm{CP} ; f, u_{0}\right)_{w}$ such that $u \in W^{1,2}(0, T ; H)$ and $\sup \psi_{w}^{t}(u(t))<+\infty$. For its detail proofs, we refer to [13, Theorems 1.1.1, 1.1.2]. $t \in[0, T]$

By Lemma 2.7, we can define a mapping $Q: E(T) \longrightarrow E(T)$ by $Q w=u$ for each $w \in E(T)$, where $u$ is a unique solution for $\left(\mathrm{CP} ; f, u_{0}\right)_{w}$. Then, we have the following property of the mapping $Q$.

Lemma 2.8 (cf. [29, Lemmas 2 and 3]). Suppose all the same conditions in Proposition 2.5. Then, there are positive constants $T_{0}, M_{0}$ and $R_{0}$ such that $Q$ is a self-mapping on $E\left(T_{0}, M_{0}, R_{0}\right)$, i.e., $Q w(=u) \in E\left(T_{0}, M_{0}, R_{0}\right)$ for any $w \in E\left(T_{0}, M_{0}, R_{0}\right)$, where

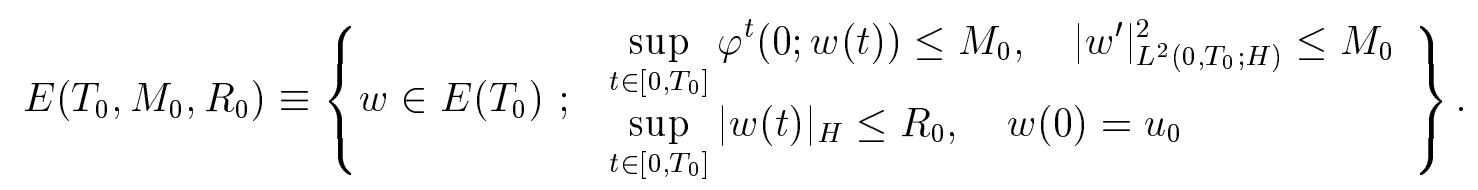

Moreover, $Q: E\left(T_{0}, M_{0}, R_{0}\right) \longrightarrow E\left(T_{0}, M_{0}, R_{0}\right)$ is continuous with respect to the topology of $C\left(\left[0, T_{0}\right] ; H\right)$. More precisely, let $\left\{w_{n}\right\} \subset E\left(T_{0}, M_{0}, R_{0}\right), w \in E\left(T_{0}, M_{0}, R_{0}\right)$, and let $u_{n}$ be the solution of $\left(\mathrm{CP} ; f, u_{0}\right)_{w_{n}}$. Suppose $w_{n} \longrightarrow w$ in $C\left(\left[0, T_{0}\right] ; H\right)$ as $n \rightarrow \infty$. Then, there is a solution $u$ of $\left(\mathrm{CP} ; f, u_{0}\right)_{w}$ on $\left[0, T_{0}\right]$ such that $u \in E\left(T_{0}, M_{0}, R_{0}\right)$ and $u_{n} \longrightarrow u$ in $C\left(\left[0, T_{0}\right] ; H\right)$ as $n \rightarrow \infty$.

Proof. Fix $R>0$ for a while and take $w \in E(T)$ with $\sup _{t \in[0, T]}|w(t)|_{H} \leq R$.

Now, we shall give a boundedness of solution $u$ to the problem $\left(\mathrm{CP} ; f, u_{0}\right)_{w}$. So, multiplying $\left(\mathrm{CP} ; f, u_{0}\right)_{w}$ by $u(t)-h(t)$, we get

$$
\begin{aligned}
& \left(u^{\prime}(t), u(t)-h(t)\right)+\varphi^{t}(w(t) ; u(t))-\varphi^{t}(w(t) ; h(t)) \\
\leq & (f(t)-G(t, w(t)), u(t)-h(t)), \quad \text { a.e. } t \in(0, T),
\end{aligned}
$$

where $h$ is the unique solution of (2.3). Taking account of $(\Phi 2),(\Phi 6)$ and (G2), we have

$$
\begin{aligned}
& \frac{d}{d t}|u(t)-h(t)|_{H}^{2}-|u(t)-h(t)|_{H}^{2} \\
\leq & N_{6}\left(|f(t)|_{H}^{2}+\left|h^{\prime}(t)\right|_{H}^{2}+\varphi^{t}(0 ; h(t))+\varphi^{t}(0 ; w(t))+R^{2}+1\right)
\end{aligned}
$$

for a positive constant $N_{6}$ dependent only on $C_{2}, C_{3}$ and $C_{4}$. By applying Gronwall's inequality to (2.11), we obtain

$$
\begin{aligned}
& \sup _{t \in[0, T]}|u(t)|_{H} \\
\leq & \sup _{t \in[0, T]}|h(t)|_{H}+e^{\frac{T}{2}} N_{6}^{\frac{1}{2}}\left\{|f|_{L^{2}(0, T ; H)}+\left|h^{\prime}\right|_{L^{2}(0, T ; H)}\right\} \\
& \quad+e^{\frac{T}{2}} N_{6}^{\frac{1}{2}} T^{\frac{1}{2}}\left\{\sup _{t \in[0, T]} \varphi^{t}(0 ; h(t))^{\frac{1}{2}}+\sup _{t \in[0, T]} \varphi^{t}(0 ; w(t))^{\frac{1}{2}}+R+1\right\} \\
\equiv & N_{7} .
\end{aligned}
$$


Next, we take the positive number $r>0$ so that $r \geq N_{7}$. Then, by Lemma 2.6 and the arguments of $\left[13\right.$, Chapter 1], we see that the function $\psi_{w}^{t}(u(t))=\varphi^{t}(w(t) ; u(t))$ satisfies the following inequality:

$$
\begin{gathered}
\psi_{w}^{t}(u(t))-\psi_{w}^{s}(u(s))+\int_{s}^{t}\left(u^{\prime}(\tau)-f(\tau)+G(\tau, w(\tau)), u^{\prime}(\tau)\right) d \tau \\
\leq \int_{s}^{t}\left|\alpha_{r}^{\prime}(\tau)\right|\left|u^{\prime}(\tau)-f(\tau)+G(\tau, w(\tau))\right|_{H}\left\{1+\psi_{w}^{\tau}(u(\tau))^{\frac{1}{2}}\right\} d \tau \\
\quad+N_{3}(1+R) \int_{s}^{t}\left[\left|\alpha_{r}^{\prime}(\tau)\right|\left\{1+\psi_{w}^{\tau}(u(\tau))\right\}+\left|w^{\prime}(\tau)\right|_{H}\left\{1+\psi_{w}^{\tau}(u(\tau))\right\}^{\frac{1}{2}}\right] d \tau
\end{gathered}
$$

for $0 \leq s \leq t \leq T$ and $w \in E(T)$ with $\sup _{t \in[0, T]}|w(t)|_{H} \leq R$.

Here, we notice the following relations:

$$
\begin{aligned}
& \left(u^{\prime}(\tau)-f(\tau)+G(\tau, w(\tau)), u^{\prime}(\tau)\right) \geq \frac{1}{2}\left|u^{\prime}(\tau)\right|_{H}^{2}-|f(\tau)|_{H}^{2}-|G(\tau, w(\tau))|_{H}^{2}, \\
& \left|\alpha_{r}^{\prime}(\tau)\right|\left|u^{\prime}(\tau)-f(\tau)+G(\tau, w(\tau))\right|_{H}\left\{1+\psi_{w}^{\tau}(u(\tau))^{\frac{1}{2}}\right\} \\
\leq & \delta\left|u^{\prime}(\tau)-f(\tau)+G(\tau, w(\tau))\right|_{H}^{2}+\delta^{-1}\left|\alpha_{r}^{\prime}(\tau)\right|^{2}\left\{1+\psi_{w}^{\tau}(u(\tau))\right\} \\
\leq & 3 \delta\left|u^{\prime}(\tau)\right|_{H}^{2}+3 \delta|f(\tau)|_{H}^{2}+3 \delta|G(\tau, w(\tau))|_{H}^{2}+\delta^{-1}\left|\alpha_{r}^{\prime}(\tau)\right|^{2}\left\{1+\psi_{w}^{\tau}(u(\tau))\right\}
\end{aligned}
$$

where we put $\delta:=\frac{1}{12}$ in $(2.15)$. Using $(2.14)-(2.15)$ in (2.13), we obtain

$$
\begin{gathered}
\psi_{w}^{t}(u(t))-\psi_{w}^{s}(u(s))+\frac{1}{4} \int_{s}^{t}\left|u^{\prime}(\tau)\right|_{H}^{2} d \tau \\
\leq N_{8}(1+R) \int_{s}^{t}\left\{X_{r}(\tau)\left\{1+\psi_{w}^{\tau}(u(\tau))\right\}+\left|w^{\prime}(\tau)\right|_{H}\left\{1+\psi_{w}^{\tau}(u(\tau))\right\}^{\frac{1}{2}}\right. \\
\left.+|G(\tau, w(\tau))|_{H}^{2}\right\} d \tau
\end{gathered}
$$

for $0 \leq s \leq t \leq T$, where the constant $N_{8}>0$ is determined only by $N_{3}$, and we put

$$
X_{r}(\tau):=|f(\tau)|_{H}^{2}+\left|\alpha_{r}^{\prime}(\tau)\right|^{2}+1 .
$$

By (థ6), (G2) and (2.16), we obtain

$$
\begin{aligned}
& \psi_{w}^{t}(u(t))-\psi_{w}^{s}(u(s))+\frac{1}{4} \int_{s}^{t}\left|u^{\prime}(\tau)\right|_{H}^{2} d \tau \\
\leq & N_{9}(1+R)^{3} \int_{s}^{t}\left\{X_{r}(\tau)+\left|w^{\prime}(\tau)\right|_{H}+\varphi^{\tau}(0 ; w(\tau))\right\}\left\{1+\psi_{w}^{\tau}(u(\tau))\right\} d \tau
\end{aligned}
$$

for $0 \leq s \leq t \leq T$, where the constant $N_{9}>0$ depends on $N_{8}, C_{2}, C_{3}$ and $C_{4}$.

Applying Gronwall's inequality to (2.17), we get

$$
\sup _{0 \leq t \leq T} \psi_{w}^{t}(u(t))+\frac{1}{4} \int_{0}^{T}\left|u^{\prime}(t)\right|_{H}^{2} d t
$$




$$
\begin{aligned}
& \leq e^{N_{9}(1+R)^{3}\left(\left|X_{r}\right|_{L^{1}(0, T)}+\left|w^{\prime}\right|_{L^{1}(0, T ; H)}+\left|\varphi^{(\cdot)}(0 ; w(\cdot))\right|_{L^{1}(0, T)}\right)} \\
& \quad \times\left\{\psi_{w}^{0}\left(u_{0}\right)+N_{9}(1+R)^{3}\left(\left|X_{r}\right|_{L^{1}(0, T)}+\left|w^{\prime}\right|_{L^{1}(0, T ; H)}+\left|\varphi^{(\cdot)}(0 ; w(\cdot))\right|_{L^{1}(0, T)}\right)\right\} .
\end{aligned}
$$

Now, we show that $Q$ is the self-mapping on $E\left(T_{0}, M_{0}, R_{0}\right)$ for some chosen constants $T_{0}>0, M_{0}>0$ and $R_{0}>0$.

Note from $(\Phi 6)$ (cf. (2.7)) that

$$
\begin{aligned}
\varphi^{t}(0 ; u(t)) & \leq 2 \varphi^{t}(w(t) ; u(t))+C_{2}^{2} R^{2}+2 C_{2} R^{2}+2 C_{2} R \\
& =2 \psi_{w}^{t}(u(t))+C_{2}^{2} R^{2}+2 C_{2} R^{2}+2 C_{2} R
\end{aligned}
$$

for any $w \in E(T)$ with $\sup _{t \in[0, T]}|w(t)|_{H} \leq R$.

Here, we take $R_{0}>0$ and $M_{0}>0$ so large that

$$
\begin{gathered}
2\left[\sup _{t \in[0, T]}|h(t)|_{H}+e^{\frac{T}{2}} N_{6}^{\frac{1}{2}}\left\{|f|_{L^{2}(0, T ; H)}+\left|h^{\prime}\right|_{L^{2}(0, T ; H)}\right\}\right] \leq R_{0}, \\
4 e^{2 N_{9}\left(1+R_{0}\right)^{3}}\left\{\varphi^{0}\left(u_{0} ; u_{0}\right)+2 N_{9}\left(1+R_{0}\right)^{3}\right\}+C_{2}^{2} R_{0}^{2}+2 C_{2} R_{0}^{2}+2 C_{2} R_{0} \leq M_{0} .
\end{gathered}
$$

Next, we choose $T_{0}>0$ so small that $T_{0} \leq T$,

$$
\begin{gathered}
\left|X_{R_{0}}\right|_{L^{1}\left(0, T_{0}\right)}=\int_{0}^{T_{0}}\left\{|f(\tau)|_{H}^{2}+\left|\alpha_{R_{0}}^{\prime}(\tau)\right|^{2}+1\right\} d \tau \leq 1, \\
\left|w^{\prime}\right|_{L^{1}\left(0, T_{0} ; H\right)}+\left|\varphi^{(\cdot)}(0 ; w(\cdot))\right|_{L^{1}\left(0, T_{0}\right)} \leq T_{0}^{\frac{1}{2}} M_{0}^{\frac{1}{2}}+T_{0} M_{0} \leq 1, \\
\sup _{t \in\left[0, T_{0}\right]}|h(t)|_{H}+e^{\frac{T_{0}}{2}} N_{6}^{\frac{1}{2}}\left\{|f|_{L^{2}\left(0, T_{0} ; H\right)}+\left|h^{\prime}\right|_{L^{2}\left(0, T_{0} ; H\right)}\right\} \\
+e^{\frac{T_{0}}{2}} N_{6}^{\frac{1}{2}} T_{0}^{\frac{1}{2}}\left\{\sup _{t \in\left[0, T_{0}\right]} \varphi^{t}(0 ; h(t))^{\frac{1}{2}}+M_{0}^{\frac{1}{2}}+R_{0}+1\right\} \leq R_{0} .
\end{gathered}
$$

Then, the estimates (2.12), (2.18) with (2.19) implies that $Q w(=u)$ belongs to the set $E\left(T_{0}, M_{0}, R_{0}\right)$ for $w \in E\left(T_{0}, M_{0}, R_{0}\right)$, thus, $Q$ is the self-mapping on $E\left(T_{0}, M_{0}, R_{0}\right)$.

Also, the continuity of $Q: E\left(T_{0}, M_{0}, R_{0}\right) \longrightarrow E\left(T_{0}, M_{0}, R_{0}\right)$ with respect to the topology of $C\left(\left[0, T_{0}\right] ; H\right)$ can be proved by a standard technique of evolution equations generated by time-dependent subdifferentials. For the detailed proof, see [29, Lemma 3] or [13, Theorem 2.7.1], for instance.

Thus, the proof of Lemma 2.8 has been completed.

[Proof of Proposition 2.5] By Lemma 2.8, we see that the self-mapping $Q: E\left(T_{0}, M_{0}\right.$, $\left.R_{0}\right) \longrightarrow E\left(T_{0}, M_{0}, R_{0}\right)$ is continuous with respect to the topology of $C\left(\left[0, T_{0}\right] ; H\right)$. Clearly, the set $E\left(T_{0}, M_{0}, R_{0}\right)$ is convex and compact in $C\left(\left[0, T_{0}\right] ; H\right)$. Therefore, the Schauder's fixed point theorem implies that the self-mapping $Q$ has a fixed point $u$ in $E\left(T_{0}, M_{0}, R_{0}\right)$, i.e. $Q u=u$. Clearly, $u$ is the solution of $\left(\mathrm{CP} ; f, u_{0}\right)$ on $\left[0, T_{0}\right]$. Thus, we construct the local solution $u$ of $\left(\mathrm{CP} ; f, u_{0}\right)$ on $\left[0, T_{0}\right]$. 
Also, by the quite standard method, we can show the global existence of solution to $\left(\mathrm{CP} ; f, u_{0}\right)$.

First, we consider the inequality (2.10). By the local existence result as above, we can take $w=u \in E\left(T_{0}\right)$ in $(2.10)$, where $u$ is the solution of (CP;f, $\left.u_{0}\right)$ on a small time interval $\left[0, T_{0}\right]$ with $0<T_{0} \leq T$. Hence, it follows from (G2) that

$$
\begin{aligned}
& \frac{d}{d t}|u(t)-h(t)|_{H}^{2}+\varphi^{t}(u(t) ; u(t)) \\
\leq & N_{10}|u(t)-h(t)|_{H}^{2}+N_{11}\left(|f(t)|_{H}^{2}+\left|h^{\prime}(t)\right|_{H}^{2}+\varphi^{t}(0 ; h(t))+1\right)
\end{aligned}
$$

for some constants $N_{10}>0$ and $N_{11}>0$ depending only on $C_{1}, C_{2}, C_{3}$ and $C_{4}$. By applying Gronwall's inequality to (2.20), we obtain

$$
\begin{aligned}
\sup _{t \in\left[0, T_{0}\right]}|u(t)|_{H} & \leq \sup _{t \in[0, T]}|h(t)|_{H}+\sqrt{N_{11} e^{N_{10} T}}\left\{|f|_{L^{2}(0, T ; H)}+\left|h^{\prime}\right|_{L^{2}(0, T ; H)}\right\} \\
& +\sqrt{N_{11} T e^{N_{10} T}}\left\{\sup _{t \in[0, T]} \varphi^{t}(0 ; h(t))^{\frac{1}{2}}+1\right\} \\
& \equiv N_{12} .
\end{aligned}
$$

Next, take a number $R>0$ so that $R \geq N_{12}$, and we now consider the inequality (2.16). Applying Schwarz inequality to the term $\left|w^{\prime}(\tau)\right|_{H}\left\{1+\psi_{w}^{\tau}(u(\tau))\right\}^{\frac{1}{2}}$ and using (G2), we get

$$
\begin{gathered}
\psi_{w}^{t}(u(t))-\psi_{w}^{s}(u(s))+\frac{1}{4} \int_{s}^{t}\left|u^{\prime}(\tau)\right|_{H}^{2} d \tau \\
\leq N_{13}(1+R)^{2} \int_{s}^{t} X_{R}(\tau)\left\{1+\psi_{w}^{\tau}(u(\tau))\right\} d \tau+\frac{1}{8} \int_{s}^{t}\left|w^{\prime}(\tau)\right|_{H}^{2} d \tau \\
+N_{14}(1+R) \int_{s}^{t} \varphi^{\tau}(w(\tau) ; w(\tau)) d \tau
\end{gathered}
$$

for $0 \leq s \leq t \leq T$, where the constants $N_{13}>0$ and $N_{14}>0$ depend on $C_{3}, C_{4}$ and $N_{8}$.

Applying Gronwall's inequality to (2.22), we obtain

$$
\begin{aligned}
\psi_{w}^{t}(u(t))+ & \frac{1}{4} \int_{0}^{t} e^{N_{13}(1+R)^{2} \int_{\tau}^{t} X_{R}(s) d s}\left|u^{\prime}(\tau)\right|_{H}^{2} d \tau \\
\leq & e^{N_{13}(1+R)^{2} \int_{0}^{T} X_{R}(s) d s}\left\{\psi_{w}^{0}\left(u_{0}\right)+N_{13}(1+R)^{2} \int_{0}^{T} X_{R}(s) d s\right\} \\
& +\frac{1}{8} \int_{0}^{t} e^{N_{13}(1+R)^{2} \int_{\tau}^{t} X_{R}(s) d s}\left|w^{\prime}(\tau)\right|_{H}^{2} d \tau \\
+ & N_{14}(1+R) \int_{0}^{t} e^{N_{13}(1+R)^{2} \int_{\tau}^{t} X_{R}(s) d s} \varphi^{\tau}(w(\tau) ; w(\tau)) d \tau
\end{aligned}
$$

Here, we take $w=u \in E\left(T_{0}\right)$ in (2.23), where $u$ is the solution of $\left(\mathrm{CP} ; f, u_{0}\right)$ on a small 
time interval $\left[0, T_{0}\right]$ with $0<T_{0} \leq T$. Then, we have

$$
\begin{gathered}
\varphi^{t}(u(t) ; u(t))+\frac{1}{8} \int_{0}^{t} e^{N_{13}(1+R)^{2} \int_{\tau}^{t} X_{R}(s) d s}\left|u^{\prime}(\tau)\right|_{H}^{2} d \tau \\
\leq N_{15}(1+R)^{2} e^{N_{16}(1+R)^{2}}\left(\varphi^{0}\left(u_{0} ; u_{0}\right)+1+\int_{0}^{t} \varphi^{\tau}(u(\tau) ; u(\tau)) d \tau\right)
\end{gathered}
$$

for $0 \leq t \leq T_{0}$, where the constants $N_{15}>0$ and $N_{16}>0$ are dependent only on the given data. By applying Gronwall's inequality to (2.24), we conclude that

$$
\begin{aligned}
& \varphi^{t}(u(t) ; u(t))+\frac{1}{8} \int_{0}^{T_{0}}\left|u^{\prime}(t)\right|_{H}^{2} d t \\
\leq & N_{17}(1+R)^{4} \exp \left(N_{18}(1+R)^{2} e^{N_{16}(1+R)^{2}}\right)\left(\varphi^{0}\left(u_{0} ; u_{0}\right)+1\right),
\end{aligned}
$$

where the constants $N_{17}>0$ and $N_{18}>0$ depend only on the given data, and are independent of $T_{0}(\leq T)$ and $R\left(\geq N_{12}\right)$.

Now, we show the global existence of solution to $\left(\mathrm{CP} ; f, u_{0}\right)$ on $[0, T]$ for any $T>0$. To do so, assume that

$$
T^{*}:=\sup \left\{T_{0} ;\left(\mathrm{CP} ; f, u_{0}\right) \text { has a solution on }\left[0, T_{0}\right]\right\}<+\infty .
$$

By the local existence result, we note $T^{*}>0$. By the definition of $T^{*}$ and the estimates (2.21) and (2.25), there is a function $u:\left[0, T^{*}\right) \rightarrow H$ such that for any $T_{0}\left(<T^{*}\right), u$ is the solution of $\left(\mathrm{CP} ; f, u_{0}\right)$ on $\left[0, T_{0}\right]$,

$$
u \in W^{1,2}\left(0, T^{*} ; H\right) \quad \text { and } \quad \varphi^{(\cdot)}(u(\cdot) ; u(\cdot)) \in L^{\infty}\left(0, T^{*}\right) .
$$

Hence, from the assumptions $(\Phi 1),(\Phi 3),(\Phi 5)$ and $(\Phi 6)$, we observe that the limit $u_{0}^{*}:=$ $\lim _{t \uparrow T^{*}} u(t)$ exists strongly in $H$ such that

$$
u_{0}^{*} \in D\left(\varphi^{T^{*}}(0 ; \cdot)\right) .
$$

Now, taking $u_{0}^{*}$ as the initial value at $t=T^{*}$, we can construct the solution $u$ beyond the time interval $\left[0, T^{*}\right]$. Therefore, we have $T^{*}=+\infty$. Hence, we conclude that the solution to $\left(\mathrm{CP} ; f, u_{0}\right)$ exists on the whole time interval $[0, T]$.

Thus, the proof of Proposition 2.5 has been completed.

To show the uniqueness of solutions to $\left(\mathrm{CP} ; f, u_{0}\right)$, we shall introduce subclasses of $\Phi\left(\left\{\alpha_{r}\right\}\right)$ and $\mathcal{G}\left(\left\{\varphi^{t}\right\}\right)$.

Definition 2.9. Let $\gamma$ be a non-negative continuous and convex function on $H$ such that $\gamma(z)+\gamma(-z)=0$ if and only if $z=0$. Then:

(i) $\left\{\varphi^{t}\right\} \in \Phi_{\gamma}\left(\left\{\alpha_{r}\right\}\right)$ if and only if $\left\{\varphi^{t}\right\} \in \Phi\left(\left\{\alpha_{r}\right\}\right)$ satisfies the following $\gamma$-accretiveness (U1) for $\varphi^{t}(\cdot ; \cdot)$ :

(U1) for any $z_{i} \in D\left(\partial \varphi^{t}\left(z_{i} ; \cdot\right)\right)$ and $z_{i}^{*} \in \partial \varphi^{t}\left(z_{i} ; z_{i}\right)(i=1,2)$, there is an element $w_{0} \in$ $\partial \gamma\left(z_{1}-z_{2}\right)$ so that $\left(z_{1}^{*}-z_{2}^{*}, w_{0}\right) \geq 0$, where $\partial \gamma$ is the subdifferential of $\gamma$ in $H$. 
(ii) $\{G(t, \cdot)\} \in \mathcal{G}_{\gamma}\left(\left\{\varphi^{t}\right\}\right)$ if and only if $\{G(t, \cdot)\} \in \mathcal{G}\left(\left\{\varphi^{t}\right\}\right)$ satisfies the following condition (U2):

(U2) for any positive number $\varepsilon>0$, there is a positive constant $C_{\varepsilon}>0$ such that

$$
\left|\left(G\left(t, z_{1}\right)-G\left(t, z_{2}\right), w_{0}\right)\right| \leq \varepsilon\left(z_{1}^{*}-z_{2}^{*}, w_{0}\right)+C_{\varepsilon}\left\{\gamma\left(z_{1}-z_{2}\right)+\gamma\left(z_{2}-z_{1}\right)\right\},
$$

whenever $\quad t \in[0, T], z_{i} \in D\left(\partial \varphi^{t}\left(z_{i} ; \cdot\right)\right), z_{i}^{*} \in \partial \varphi^{t}\left(z_{i} ; z_{i}\right)(i=1,2)$, and $w_{0} \in \partial \gamma\left(z_{1}-z_{2}\right)$ with $\left(z_{1}^{*}-z_{2}^{*}, w_{0}\right)_{H} \geq 0$.

By the same proof of [29, Theorem 3], we can show the following result of the uniqueness of solutions to $\left(\mathrm{CP} ; f, u_{0}\right)$. For the detailed proof, see [29, Theorem 3].

Proposition 2.10 (cf. [29, Theorem 3]). Let T be any positive number. Assume $\left\{\varphi^{t}\right\} \in$ $\Phi_{\gamma}\left(\left\{\alpha_{r}\right\}\right)$ and $\{G(t, \cdot)\} \in \mathcal{G}_{\gamma}\left(\left\{\varphi^{t}\right\}\right)$. Then, for each $f \in L^{2}(0, T ; H)$ and $u_{0} \in D\left(\varphi^{0}(0 ; \cdot)\right)$, the solution $u$ of $\left(\mathrm{CP} ; f, u_{0}\right)$ is unique.

\section{Main results}

In this section, we state the main results in this paper.

We begin by recalling a notion of convergence for convex functions, developed by Mosco [20].

Definition 3.1 (cf. [20]). Let $\psi, \psi_{n}(n \in \mathbb{N})$ be proper, l.s.c. and convex functions on a Hilbert space $H$. Then, we say that $\psi_{n}$ converges to $\psi$ on $H$ in the sense of Mosco [20] as $n \rightarrow \infty$, if the following two conditions are satisfied:

(i) for any subsequence $\left\{\psi_{n_{k}}\right\} \subset\left\{\psi_{n}\right\}$, if $z_{k} \rightarrow z$ weakly in $H$ as $k \rightarrow \infty$, then

$$
\liminf _{k \rightarrow \infty} \psi_{n_{k}}\left(z_{k}\right) \geq \psi(z)
$$

(ii) for any $z \in D(\psi)$, there is a sequence $\left\{z_{n}\right\}$ in $H$ such that

$$
z_{n} \longrightarrow z \text { in } H \text { as } n \rightarrow \infty \quad \text { and } \quad \lim _{n \rightarrow \infty} \psi_{n}\left(z_{n}\right)=\psi(z) .
$$

Now, we state the first main result in this paper, which is concerned with the convergence of solutions for $(\mathrm{CP})$ and $(\mathrm{CP})_{n}$.

Theorem 3.2. Let $0<T<+\infty$ be fixed. Assume $\left\{\varphi^{t}\right\} \in \Phi_{\gamma}\left(\left\{\alpha_{r}\right\}\right),\{G(t, \cdot)\} \in$ $\mathcal{G}_{\gamma}\left(\left\{\varphi^{t}\right\}\right), f \in L^{2}(0, T ; H)$ and $u_{0} \in D\left(\varphi^{0}(0 ; \cdot)\right)$. Also, suppose $\left\{\varphi_{n}^{t}\right\} \in \Phi_{\gamma}\left(\left\{\alpha_{r}\right\}\right)$, $\left\{G_{n}(t, \cdot)\right\} \in \mathcal{G}_{\gamma}\left(\left\{\varphi_{n}^{t}\right\}\right),\left\{f_{n}\right\} \subset L^{2}(0, T ; H)$ and $u_{0, n} \in D\left(\varphi_{n}^{0}(0 ; \cdot)\right)$ for $n=1,2, \cdots$. Furthermore, assume the following convergence conditions:

(K1) $\varphi_{n}^{t}(w ; \cdot)$ converges to $\varphi^{t}(w ; \cdot)$ on $H$ in the sense of Mosco [20] for each $t \in[0, T$ ] and $w \in H($ as $n \rightarrow \infty)$. Moreover, $\bigcup_{n=1}^{\infty}\left\{z \in H ; \varphi_{n}^{t}(w ; z) \leq k\right\}$ is relatively compact in $H$ for all $k>0, t \in[0, T]$ and $w \in H$, where $\left\{\varphi_{\infty}^{t}\right\}=\left\{\varphi^{t}\right\} \in \Phi_{\gamma}\left(\left\{\alpha_{r}\right\}\right)$ when $n=\infty$; 
$(\mathrm{K} 2) G_{n}\left(t_{n}, z_{n}\right) \rightarrow G(t, z)$ weakly in $H$ as $n \rightarrow \infty$, if $\left\{t_{n}\right\} \subset[0, T],\left\{z_{n}\right\} \subset H, t_{n} \rightarrow t$, $z_{n} \rightarrow z$ in $H($ as $n \rightarrow \infty)$ and $\left\{\varphi_{n}^{t_{n}}\left(z_{n} ; z_{n}\right)\right\}$ is bounded;

(K3) $u_{0, n} \rightarrow u_{0}$ in $H$ as $n \rightarrow \infty$, and $\left\{\varphi_{n}^{0}\left(u_{0, n} ; u_{0, n}\right)\right\}$ is bounded;

(K4) $f_{n} \rightarrow f$ weakly in $L^{2}(0, T ; H)$ as $n \rightarrow \infty$.

Then, the unique solution $u_{n}$ of $\left(\mathrm{CP} ; f_{n}, u_{0, n}\right)_{n}$ converges to one $u$ of $\left(\mathrm{CP} ; f, u_{0}\right)$ in the following sense:

$$
u_{n} \rightarrow u \text { in } C([0, T] ; H), \quad u_{n}^{\prime} \rightarrow u^{\prime} \text { weakly in } L^{2}(0, T ; H) \quad \text { as } n \rightarrow \infty .
$$

The proof of Theorem 3.2 is given in Section 4 by using Mosco convergence.

Next, we state the second main result in this paper, which is concerned with the existence of an optimal control for (OP).

Theorem 3.3. Assume $\left\{\varphi^{t}\right\} \in \Phi_{\gamma}\left(\left\{\alpha_{r}\right\}\right),\{G(t, \cdot)\} \in \mathcal{G}_{\gamma}\left(\left\{\varphi^{t}\right\}\right), u_{0} \in D\left(\varphi^{0}(0 ; \cdot)\right)$ and $u_{d} \in$ $L^{2}(0, T ; H)$. Then, the problem $(\mathrm{OP})$ has at least one optimal control $f^{*} \in L^{2}(0, T ; H)$ so that

$$
J\left(f^{*}\right)=\inf _{f \in L^{2}(0, T ; H)} J(f),
$$

where $J(\cdot)$ is the cost functional given in (1.4).

The proof of Theorem 3.3 is given in Section 5 by using the result (cf. Corollary 4.1 below) of convergence of solutions to the state problem (CP; $\left.f, u_{0}\right)$.

Also, let us study the control problem $(\mathrm{OP})_{1, n}$ for each $n \in \mathbb{N}$. Then, we have the following third main result in this paper, which is concerned with the relationship between problems $(\mathrm{OP})$ and $(\mathrm{OP})_{1, n}(n \in \mathbb{N})$.

Theorem 3.4. Assume $u_{d} \in L^{2}(0, T ; H),\left\{\varphi_{n}^{t}\right\} \in \Phi_{\gamma}\left(\left\{\alpha_{r}\right\}\right),\left\{G_{n}(t, \cdot)\right\} \in \mathcal{G}_{\gamma}\left(\left\{\varphi_{n}^{t}\right\}\right)$ and $u_{0, n} \in D\left(\varphi_{n}^{0}(0 ; \cdot)\right)$ for $n=1,2, \cdots$. Then, for each $n \in \mathbb{N}$, the problem $(\mathrm{OP})_{1, n}$ has at least one optimal control $f_{1, n}^{*} \in L^{2}(0, T ; H)$ so that

$$
J_{1, n}\left(f_{1, n}^{*}\right)=\inf _{f \in L^{2}(0, T ; H)} J_{1, n}(f),
$$

where $J_{1, n}(\cdot)$ is the cost functional given in $(1.5)$.

Furthermore, suppose $\left\{\varphi^{t}\right\} \in \Phi_{\gamma}\left(\left\{\alpha_{r}\right\}\right),\{G(t, \cdot)\} \in \mathcal{G}_{\gamma}\left(\left\{\varphi^{t}\right\}\right), u_{0} \in D\left(\varphi^{0}(0 ; \cdot)\right)$ and the convergence conditions (K1), (K2) and (K3) in Theorem 3.2. Then, there is a subsequence $\left\{n_{k}\right\} \subset\{n\}$ and a function $f^{* *} \in L^{2}(0, T ; H)$ such that $f^{* *}$ is the optimal control of $(\mathrm{OP})$, $n_{k} \rightarrow \infty$, and

$$
f_{1, n_{k}}^{*} \rightarrow f^{* *} \quad \text { weakly in } L^{2}(0, T ; H) \text { as } k \rightarrow \infty \text {. }
$$

In order to show the strong convergence of optimal controls, we consider the another type of control problems $(\mathrm{OP})_{2, n}$ for each $n \in \mathbb{N}$, which is introduced in Section 1.

Now, we mention our final main result in this paper, which is concerned with the relationship between problems $(\mathrm{OP})$ and $(\mathrm{OP})_{2, n}(n \in \mathbb{N})$. 
Theorem 3.5. Assume $u_{d} \in L^{2}(0, T ; H),\left\{\varphi_{n}^{t}\right\} \in \Phi_{\gamma}\left(\left\{\alpha_{r}\right\}\right),\left\{G_{n}(t, \cdot)\right\} \in \mathcal{G}_{\gamma}\left(\left\{\varphi_{n}^{t}\right\}\right)$ and $u_{0, n} \in D\left(\varphi_{n}^{0}(0 ; \cdot)\right)$ for $n=1,2, \cdots$. Let $\left\{\varphi^{t}\right\} \in \Phi_{\gamma}\left(\left\{\alpha_{r}\right\}\right),\{G(t, \cdot)\} \in \mathcal{G}_{\gamma}\left(\left\{\varphi^{t}\right\}\right), u_{0} \in$ $D\left(\varphi^{0}(0 ; \cdot)\right)$, and let $f^{*}$ be the optimal control obtained in Theorem 3.3. Then, for each $n \in \mathbb{N}$, the problem $(\mathrm{OP})_{2, n}$ has at least one optimal control $f_{2, n}^{*} \in L^{2}(0, T ; H)$ so that

$$
J_{2, n}\left(f_{2, n}^{*}\right)=\inf _{f \in L^{2}(0, T ; H)} J_{2, n}(f),
$$

where $J_{2, n}(\cdot)$ is the cost functional given in (1.6).

Furthermore, suppose the convergence conditions (K1), (K2) and (K3) in Theorem 3.2. Let $u_{n}^{*}$ and $u^{*}$ be unique solutions of $\left(\mathrm{CP} ; f_{2, n}^{*}, u_{0, n}\right)_{n}$ and $\left(\mathrm{CP} ; f^{*}, u_{0}\right)$ on $[0, T]$, respectively. Then, there is a subsequence $\left\{n_{k}\right\} \subset\{n\}$ such that $n_{k} \rightarrow \infty$,

$$
f_{2, n_{k}}^{*} \longrightarrow f^{*} \quad \text { strongly in } L^{2}(0, T ; H)
$$

and

$$
u_{n_{k}}^{*} \longrightarrow u^{*} \quad \text { strongly in } C([0, T] ; H)
$$

as $k \rightarrow \infty$.

The proof of Theorems 3.4 and 3.5 is given in Section 5. Roughly summarized, the convergences (3.1)-(3.3) are proved by using the result (Theorem 3.2) of the continuous dependence between solutions of $\left(\mathrm{CP} ; f, u_{0}\right)$ and $\left(\mathrm{CP} ; f, u_{0, n}\right)_{n}$.

\section{Convergence of solutions}

In this section, we prove Theorem 3.2 concerning the convergence of solutions to $(\mathrm{CP})$ and $(\mathrm{CP})_{n}(n \in \mathbb{N})$.

We begin by the key lemma to showing Theorem 3.2.

Lemma 4.1 (cf. [13, Proposition 2.7.1]). Let $\left\{\varphi_{n}^{t}\right\} \in \Phi\left(\left\{\alpha_{r}\right\}\right)$ and $\left\{\varphi^{t}\right\} \in \Phi\left(\left\{\alpha_{r}\right\}\right)$. For each $w \in L^{2}(0, T ; H)$, put

$$
\begin{array}{r}
\Phi_{n}^{w}(z):=\int_{0}^{T} \varphi_{n}^{t}(w(t) ; z(t)) d t \quad \text { and } \quad \Phi^{w}(z):=\int_{0}^{T} \varphi^{t}(w(t) ; z(t)) d t \\
\text { for any } z \in L^{2}(0, T ; H) .
\end{array}
$$

Then, $\Phi_{n}^{w}$, $\Phi^{w}$ are proper, l.s.c. and convex on $L^{2}(0, T ; H)$. Moreover, assume the condition (K1) in Theorem 3.2, and $w \in W^{1,2}(0, T ; H)$. Then,

$$
\Phi_{n}^{w} \text { converges to } \Phi^{w} \text { on } L^{2}(0, T ; H) \text { in the sense of Mosco [20] }
$$

as $n \rightarrow \infty$.

Proof. Assume $w \in L^{2}(0, T ; H)$. Then, clearly, $\Phi_{n}^{w}$ and $\Phi^{w}$ are proper, 1.s.c. and convex on $L^{2}(0, T ; H)$, since $\varphi_{n}^{t}(w(t) ; \cdot)$ and $\varphi^{t}(w(t) ; \cdot)$ are proper, l.s.c. and convex on $H$ (cf. $(\Phi 1)$ in Definition 2.2). 
Next, assume $w \in W^{1,2}(0, T ; H)$. Then, we see from Lemma 2.6 that $\psi_{w}^{t}(\cdot)=$ $\varphi^{t}(w(t) ; \cdot)$ satisfies the time-dependence condition proposed by Kenmochi [13]. Thus, by applying the abstract result of [13, Proposition 2.7.1], we conclude that the convergence (4.1) holds. For the detailed proof, we refer to [13, Proposition 2.7.1].

Now, we show the first main result in this paper, which is concerned with the convergence of solutions for $(\mathrm{CP})$ and $(\mathrm{CP})_{n}$.

[Proof of Theorem 3.2] Let $u_{n}$ be the unique solution of $\left(\mathrm{CP} ; f_{n}, u_{0, n}\right)_{n}$. Note from the assumptions (K3) and (K4) that $\left\{u_{0, n}\right\}$ is bounded in $H,\left\{\varphi_{n}^{0}\left(u_{0, n} ; u_{0, n}\right)\right\}$ is bounded, and $\left\{f_{n}\right\}$ is bounded in $L^{2}(0, T ; H)$. Therefore, by the estimates $(2.1)$ and $(2.2)$, we see that

$$
\left\{u_{n}\right\} \text { is bounded in } W^{1,2}(0, T ; H)
$$

and

$$
\sup _{t \in[0, T]} \varphi_{n}^{t}\left(u_{n}(t) ; u_{n}(t)\right) \text { is bounded. }
$$

From the assumption of the level set compactness (cf. (K1)) we observe that there is a subsequence $\left\{n_{k}\right\} \subset\{n\}$ and a function $u \in W^{1,2}(0, T ; H)$ satisfying

$$
\begin{array}{ll}
u_{n_{k}} \longrightarrow u & \text { strongly in } C([0, T] ; H), \\
u_{n_{k}}^{\prime} \rightarrow u^{\prime} & \text { weakly in } L^{2}(0, T ; H)
\end{array}
$$

as $k \rightarrow \infty$.

Now, we show that the function $u$ is the solution to (CP; $\left.f, u_{0}\right)$.

Since $u \in W^{1,2}(0, T ; H)$, we infer from Lemma 4.1 that

$$
\Phi_{n}^{u}(\cdot):=\int_{0}^{T} \varphi_{n}^{t}(u(t) ; \cdot) d t \quad \text { converges to } \quad \Phi^{u}(\cdot):=\int_{0}^{T} \varphi^{t}(u(t) ; \cdot) d t
$$

on $L^{2}(0, T ; H)$ in the sense of Mosco [20] as $n \rightarrow \infty$. Therefore, for any $w \in D\left(\Phi^{u}\right)$, there is a sequence $\left\{w_{n}\right\}$ in $L^{2}(0, T ; H)$ such that

$$
w_{n} \longrightarrow w \text { in } L^{2}(0, T ; H) \text { as } n \rightarrow \infty \quad \text { and } \quad \lim _{n \rightarrow \infty} \Phi_{n}^{u}\left(w_{n}\right)=\Phi^{u}(w) .
$$

Now, we observe from $(\Phi 6)$ that

$$
\begin{gathered}
\quad\left|\int_{0}^{T} \varphi_{n_{k}}^{t}\left(u_{n_{k}}(t) ; w_{n_{k}}(t)\right) d t-\int_{0}^{T} \varphi^{t}(u(t) ; w(t)) d t\right| \\
\leq\left|\int_{0}^{T} \varphi_{n_{k}}^{t}\left(u_{n_{k}}(t) ; w_{n_{k}}(t)\right) d t-\int_{0}^{T} \varphi_{n_{k}}^{t}\left(u(t) ; w_{n_{k}}(t)\right) d t\right| \\
\quad+\left|\int_{0}^{T} \varphi_{n_{k}}^{t}\left(u(t) ; w_{n_{k}}(t)\right) d t-\int_{0}^{T} \varphi^{t}(u(t) ; w(t)) d t\right| \\
\leq \quad \int_{0}^{T} C_{2}\left|u_{n_{k}}(t)-u(t)\right|_{H}\left(\varphi_{n_{k}}^{t}\left(0 ; w_{n_{k}}(t)\right)^{\frac{1}{2}}+\left|u_{n_{k}}(t)\right|_{H}+|u(t)|_{H}+1\right) d t \\
\quad+\left|\Phi_{n_{k}}^{u}\left(w_{n_{k}}\right)-\Phi^{u}(w)\right|
\end{gathered}
$$




$$
\begin{gathered}
\leq C_{2} T^{\frac{1}{2}}\left|u_{n_{k}}-u\right|_{C([0, T] ; H)}\left\{\left(\int_{0}^{T} \varphi_{n_{k}}^{t}\left(0 ; w_{n_{k}}(t)\right) d t\right)^{\frac{1}{2}}+\left|u_{n_{k}}\right|_{L^{2}(0, T ; H)}\right. \\
\left.+|u|_{L^{2}(0, T ; H)}+T^{\frac{1}{2}}\right\} \\
+\left|\Phi_{n_{k}}^{u}\left(w_{n_{k}}\right)-\Phi^{u}(w)\right| .
\end{gathered}
$$

Here, we note from (4.6) and (\$6) (cf. (2.7)) that

$$
\int_{0}^{T} \varphi_{n_{k}}^{t}\left(0 ; w_{n_{k}}(t)\right) d t \quad \text { is bounded uniformly in } n_{k} .
$$

Thus, by (4.2), (4.4), (4.6), (4.7) and (4.8), we have

$$
\int_{0}^{T} \varphi_{n_{k}}^{t}\left(u_{n_{k}}(t) ; w_{n_{k}}(t)\right) d t \longrightarrow \int_{0}^{T} \varphi^{t}(u(t) ; w(t)) d t \quad \text { as } k \rightarrow \infty .
$$

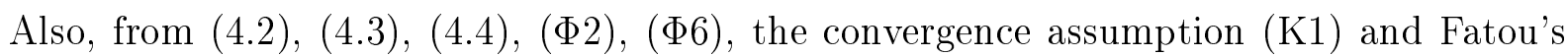
lemma, it follows that

$$
\begin{aligned}
& \liminf _{k \rightarrow \infty} \int_{0}^{T} \varphi_{n_{k}}^{t}\left(u_{n_{k}}(t) ; u_{n_{k}}(t)\right) d t \\
& \geq \liminf _{k \rightarrow \infty}\left\{\int_{0}^{T} \varphi_{n_{k}}^{t}\left(u_{n_{k}}(t) ; u_{n_{k}}(t)\right) d t-\int_{0}^{T} \varphi_{n_{k}}^{t}\left(u(t) ; u_{n_{k}}(t)\right) d t\right\} \\
& \quad+\liminf _{k \rightarrow \infty} \int_{0}^{T} \varphi_{n_{k}}^{t}\left(u(t) ; u_{n_{k}}(t)\right) d t \\
& \geq \int_{0}^{T} \liminf _{k \rightarrow \infty} \varphi_{n_{k}}^{t}\left(u(t) ; u_{n_{k}}(t)\right) d t \\
& \geq \int_{0}^{T} \varphi^{t}(u(t) ; u(t)) d t .
\end{aligned}
$$

Now, let us show that the function $u$ is the solution to (CP; $\left.f, u_{0}\right)$. Since $u_{n_{k}}$ is the solution of $\left(\mathrm{CP} ; f_{n_{k}}, u_{0, n_{k}}\right)_{n_{k}}$, then, the following inequality holds:

$$
\begin{aligned}
& \int_{0}^{T}\left(f_{n_{k}}(t)-u_{n_{k}}^{\prime}(t)-G_{n_{k}}\left(t, u_{n_{k}}(t)\right), w_{n_{k}}(t)-u_{n_{k}}(t)\right) d t \\
\leq & \int_{0}^{T} \varphi_{n_{k}}^{t}\left(u_{n_{k}}(t) ; w_{n_{k}}(t)\right) d t-\int_{0}^{T} \varphi_{n_{k}}^{t}\left(u_{n_{k}}(t) ; u_{n_{k}}(t)\right) d t
\end{aligned}
$$

and

$$
u_{n_{k}}(0)=u_{0, n_{k}}
$$

where $\left\{w_{n_{k}}\right\}$ is a sequence in $L^{2}(0, T ; H)$ obtained in (4.6).

Taking account of (K1)-(K4), (4.3)-(4.6), (4.9)-(4.10), and letting $k \rightarrow \infty$ in (4.11)(4.12), we get

$$
\begin{aligned}
& \int_{0}^{T}\left(f(t)-u^{\prime}(t)-G(t, u(t)), w(t)-u(t)\right) d t \\
\leq & \int_{0}^{T} \varphi^{t}(u(t) ; w(t)) d t-\int_{0}^{T} \varphi^{t}(u(t) ; u(t)) d t \\
= & \Phi^{u}(w)-\Phi^{u}(u)
\end{aligned}
$$


and

$$
u(0)=u_{0} .
$$

Therefore, it follows from (4.13) that $f-u^{\prime}-G(\cdot, u) \in \partial \Phi^{u}(u)$ in $L^{2}(0, T ; H)$, since $w$ is the arbitrary function in $D\left(\Phi^{u}\right)$. Here, note from $u \in W^{1,2}(0, T ; H)$ that we can get the standard time-dependence condition of $\psi_{u}^{t}(\cdot):=\varphi^{t}(u(t) ; \cdot)$ (cf. Lemma 2.6). Therefore, by taking account of [12, Proposition 1.1 and Lemma 3.3], we infer from (4.13) that $f(t)-u^{\prime}(t)-G(t, u(t)) \in \partial \varphi^{t}(u(t) ; u(t))$ for a.e. $t \in[0, T]$. Hence, $u$ is the solution of $\left(\mathrm{CP} ; f, u_{0}\right)$ on $[0, T]$.

By the uniqueness of solutions to $\left(\mathrm{CP} ; f, u_{0}\right)$, we conclude that the solution $u_{n}$ of $\left(\mathrm{CP} ; f_{n}, u_{0, n}\right)_{n}$ converges to one $u$ of $\left(\mathrm{CP} ; f, u_{0}\right)$ in the following sense:

$$
u_{n} \rightarrow u \text { in } C([0, T] ; H), \quad u_{n}^{\prime} \rightarrow u^{\prime} \text { weakly in } L^{2}(0, T ; H) \quad \text { as } n \rightarrow \infty .
$$

Thus, the proof of Theorem 3.2 has been completed.

We can easily get the following result of the convergence of solutions to $(\mathrm{CP})$.

Corollary 4.1. Let $0<T<+\infty$ be fixed. Assume $\left\{\varphi^{t}\right\} \in \Phi_{\gamma}\left(\left\{\alpha_{r}\right\}\right)$ and $\{G(t, \cdot)\} \in$ $\mathcal{G}_{\gamma}\left(\left\{\varphi^{t}\right\}\right)$. Also, suppose $\left\{f_{m}\right\} \subset L^{2}(0, T ; H)$ and $\left\{u_{0, m}\right\} \subset D\left(\varphi^{0}(0 ; \cdot)\right)$ so that $\left\{\varphi^{0}\left(u_{0, m} ; u_{0, m}\right)\right\}$ is bounded,

$$
f_{m} \rightarrow f \text { weakly in } L^{2}(0, T ; H) \text { and } u_{0, m} \rightarrow u_{0} \text { in } H \text { as } m \rightarrow \infty
$$

for some $f \in L^{2}(0, T ; H)$ and $u_{0} \in D\left(\varphi^{0}(0 ; \cdot)\right)$. Then, the unique solution $u_{m}$ of $\left(\mathrm{CP} ; f_{m}, u_{0, m}\right)$ converges to one $u$ of $\left(\mathrm{CP} ; f, u_{0}\right)$ in the following sense:

$$
u_{m} \rightarrow u \text { in } C([0, T] ; H), \quad u_{m}^{\prime} \rightarrow u^{\prime} \text { weakly in } L^{2}(0, T ; H) \quad \text { as } m \rightarrow \infty .
$$

Also, we get the convergence result of solutions to $(\mathrm{CP})_{n}$, but, we don't mention the detailed statements.

\section{Optimal control problems}

In this section, we study the optimal control problem (OP). Also, we consider the optimal control problems $(\mathrm{OP})_{1, n}$ and $(\mathrm{OP})_{2, n}(n \in \mathbb{N})$. Moreover, by using the convergence result (Theorem 3.2) of solutions to $\left(\mathrm{CP} ; f, u_{0}\right)$ and $\left(\mathrm{CP} ; f, u_{0, n}\right)_{n}$, we prove Theorem 3.4 (resp. Theorem 3.5) concerned with the relationship between (OP) and (OP) ${ }_{1, n}$ (resp. (OP) and $\left.(\mathrm{OP})_{2, n}\right)$.

At first, we prove Theorem 3.3, which is concerned with the existence of an optimal control for (OP).

[Proof of Theorem 3.3] By the quite standard method, we can prove Theorem 3.3. So, let $\left\{f_{n}\right\} \subset L^{2}(0, T ; H)$ be a minimizing sequence such that

$$
\lim _{n \rightarrow \infty} J\left(f_{n}\right)=\inf _{f \in L^{2}(0, T ; H)} J(f)
$$


Then, by the definition (1.4) of $J(\cdot)$, we see that $\left\{f_{n}\right\}$ is bounded in $L^{2}(0, T ; H)$. Hence, there is a subsequence $\left\{n_{k}\right\} \subset\{n\}$ and a function $f^{*} \in L^{2}(0, T ; H)$ such that $n_{k} \rightarrow \infty$ and

$$
f_{n_{k}} \rightarrow f^{*} \text { weakly in } L^{2}(0, T ; H) \quad \text { as } k \rightarrow \infty .
$$

Here, for any $k \in \mathbb{N}$, let $u_{n_{k}}$ be a unique solution to $\left(\mathrm{CP} ; f_{n_{k}}, u_{0}\right)$ on $[0, T]$. Then, from (5.1) and Corollary 4.1, we observe that

$$
u_{n_{k}} \longrightarrow u^{*} \quad \text { strongly in } C([0, T] ; H) \text { as } k \rightarrow \infty,
$$

where $u^{*}$ is a unique solution to $\left(\mathrm{CP} ; f^{*}, u_{0}\right)$ on $[0, T]$.

Hence, it follows from (5.1)-(5.2) and the weak lower semicontinuity of $L^{2}$-norm that

$$
J\left(f^{*}\right) \leq \lim _{k \rightarrow \infty} J\left(f_{n_{k}}\right)=\inf _{f \in L^{2}(0, T ; H)} J(f) .
$$

The above inequality implies that $f^{*} \in L^{2}(0, T ; H)$ is an optimal control for (OP). Thus, the proof of Theorem 3.3 has been completed.

Now, let us prove Theorem 3.4, which is concerned with the relationship between (OP) and $(\mathrm{OP})_{1, n}(n \in \mathbb{N})$.

[Proof of Theorem 3.4] By replacing $\varphi^{t}(\cdot ; \cdot)(\operatorname{resp} . G(t, \cdot))$ with $\varphi_{n}^{t}(\cdot ; \cdot)\left(\operatorname{resp} . G_{n}(t, \cdot)\right)$ in Corollary 4.1, we get the result of convergence of solutions for $(\mathrm{CP})_{n}$. Thus, for each $n \in \mathbb{N}$, by the same proof of Theorem 3.3, we can show the existence of an optimal control $f_{1, n}^{*}$ of $(\mathrm{OP})_{1, n}$ such that

$$
J_{1, n}\left(f_{1, n}^{*}\right)=\inf _{f \in L^{2}(0, T ; H)} J_{1, n}(f),
$$

where $J_{1, n}(\cdot)$ is the cost functional defined in $(1.5)$.

Now, we show (3.1). Let $f$ be any function in $L^{2}(0, T ; H)$. Also, let $u_{n}$ be a unique solution for $\left(\mathrm{CP} ; f, u_{0, n}\right)_{n}$ on $[0, T]$, and let $u$ be a unique solution for $\left(\mathrm{CP} ; f, u_{0}\right)$ on $[0, T]$. Then, we observe from the assumptions (K1)-(K3) and Theorem 3.2 that

$$
u_{n} \longrightarrow u \quad \text { strongly in } C([0, T] ; H) \text { as } n \rightarrow \infty \text {. }
$$

Since $f_{1, n}^{*}$ is the optimal control of $(\mathrm{OP})_{1, n}$, we see that

$$
J_{1, n}\left(f_{1, n}^{*}\right) \leq J_{1, n}(f)=\frac{1}{2} \int_{0}^{T}\left|\left(u_{n}-u_{d}\right)(t)\right|_{H}^{2} d t+\frac{1}{2} \int_{0}^{T}|f(t)|_{H}^{2} d t .
$$

Clearly, it follows from (1.5), (5.3)-(5.4) that $\left\{f_{1, n}^{*}\right\}$ is bounded in $L^{2}(0, T ; H)$ with respect to $n \in \mathbb{N}$. Thus, there is a subsequence $\left\{n_{k}\right\} \subset\{n\}$ and a function $f^{* *} \in L^{2}(0, T ; H)$ such that $n_{k} \rightarrow \infty$ and

$$
f_{1, n_{k}}^{*} \rightarrow f^{* *} \quad \text { weakly in } L^{2}(0, T ; H) \quad \text { as } k \rightarrow \infty .
$$

For any $k \in \mathbb{N}$, let $u_{n_{k}}^{*}$ be a unique solution of $\left(\mathrm{CP} ; f_{1, n_{k}}^{*}, u_{0, n_{k}}\right)_{n_{k}}$ on $[0, T]$. Then, by (5.5), the assumptions (K1)-(K3) and Theorem 3.2, we see that $u_{n_{k}}^{*}$ converges to the unique solution $u^{* *}$ of $\left(\mathrm{CP} ; f^{* *}, u_{0}\right)$ on $[0, T]$ in the sense that

$$
u_{n_{k}}^{*} \longrightarrow u^{* *} \quad \text { strongly in } C([0, T] ; H) \quad \text { as } k \rightarrow \infty .
$$


Now, by using (5.3)-(5.6) and the weak lower semicontinuity of $L^{2}$-norm, we see that

$$
J\left(f^{* *}\right) \leq \liminf _{k \rightarrow \infty} J_{1, n_{k}}\left(f_{1, n_{k}}^{*}\right) \leq J(f) .
$$

Since $f$ is any function in $L^{2}(0, T ; H)$, we infer from the above inequality that $f^{* *}$ is the optimal control of (OP) satisfying the convergence (5.5) (i.e. (3.1)). Thus, the proof of Theorem 3.4 has been completed.

Finally, by using Theorem 3.2, we show Theorem 3.5, which is concerned with the relationship between $(\mathrm{OP})$ and $(\mathrm{OP})_{2, n}(n \in \mathbb{N})$.

[Proof of Theorem 3.5] At first, note that by the same argument in Theorem 3.3, namely, by using the result of the continuous dependence of solutions for $(\mathrm{CP})_{n}$ (cf. Corollary 4.1), we can get the existence of an optimal control $f_{2, n}^{*}$ of $(\mathrm{OP})_{2, n}$ for each $n \in \mathbb{N}$, such that

$$
J_{2, n}\left(f_{2, n}^{*}\right)=\inf _{f \in L^{2}(0, T ; H)} J_{2, n}(f),
$$

where $J_{2, n}(\cdot)$ is the cost functional defined in (1.6).

Now, we show (3.2)-(3.3). For the optimal control $f^{*}$ of (OP) obtained in Theorem 3.3 , let $u_{n}$ be a unique solution to $\left(\mathrm{CP} ; f^{*}, u_{0, n}\right)_{n}$ on $[0, T]$, and let $u^{*}$ be a unique solution to $\left(\mathrm{CP} ; f^{*}, u_{0}\right)$ on $[0, T]$. Then, we observe from the assumptions (K1)-(K3) and Theorem 3.2 that

$$
u_{n} \longrightarrow u^{*} \quad \text { strongly in } C([0, T] ; H) \text { as } n \rightarrow \infty .
$$

On the other hand, since $f_{2, n}^{*}$ is the optimal control of $(\mathrm{OP})_{2, n}$, we see that

$$
J_{2, n}\left(f_{2, n}^{*}\right) \leq J_{2, n}\left(f^{*}\right)=\frac{1}{2} \int_{0}^{T}\left|\left(u_{n}-u_{d}\right)(t)\right|_{H}^{2} d t+\frac{1}{2} \int_{0}^{T}\left|f^{*}(t)\right|_{H}^{2} d t .
$$

Clearly, it follows from (1.6) and $(5.7)-(5.8)$ that $\left\{f_{2, n}^{*}\right\}$ is bounded in $L^{2}(0, T ; H)$ with respect to $n \in \mathbb{N}$. Thus, there is a subsequence $\left\{n_{k}\right\} \subset\{n\}$ and a function $f^{\circ} \in L^{2}(0, T ; H)$ such that $n_{k} \rightarrow \infty$ and

$$
f_{2, n_{k}}^{*} \rightarrow f^{\circ} \quad \text { weakly in } L^{2}(0, T ; H) \quad \text { as } k \rightarrow \infty .
$$

For any $k \in \mathbb{N}$, let $u_{n_{k}}^{*}$ be a unique solution of $\left(\mathrm{CP} ; f_{2, n_{k}}^{*}, u_{0, n_{k}}\right)_{n_{k}}$ on $[0, T]$. Then, by the assumptions (K1)-(K3), (5.9) and Theorem 3.2, we see that $u_{n_{k}}^{*}$ converges to the unique solution $u^{\circ}$ of $\left(\mathrm{CP} ; f^{\circ}, u_{0}\right)$ on $[0, T]$ in the sense that

$$
u_{n_{k}}^{*} \longrightarrow u^{\circ} \quad \text { strongly in } C([0, T] ; H) \quad \text { as } k \rightarrow \infty .
$$

Now, by using (5.7)-(5.10) and the weak lower semicontinuity of $L^{2}$-norm, we see that

$$
\begin{aligned}
& \frac{1}{2} \limsup _{k \rightarrow \infty} \int_{0}^{T}\left|\left(f_{2, n_{k}}^{*}-f^{*}\right)(t)\right|_{H}^{2} d t \\
\leq & \limsup _{k \rightarrow \infty}\left(J_{2, n_{k}}\left(f^{*}\right)-\frac{1}{2} \int_{0}^{T}\left|\left(u_{n_{k}}^{*}-u_{d}\right)(t)\right|_{H}^{2} d t-\frac{1}{2} \int_{0}^{T}\left|f_{2, n_{k}}^{*}(t)\right|_{H}^{2} d t\right) \\
\leq & \frac{1}{2} \int_{0}^{T}\left|\left(u^{*}-u_{d}\right)(t)\right|_{H}^{2} d t+\frac{1}{2} \int_{0}^{T}\left|f^{*}(t)\right|_{H}^{2} d t
\end{aligned}
$$




$$
\begin{aligned}
& -\frac{1}{2} \liminf _{k \rightarrow \infty} \int_{0}^{T}\left|\left(u_{n_{k}}^{*}-u_{d}\right)(t)\right|_{H}^{2} d t-\frac{1}{2} \liminf _{k \rightarrow \infty} \int_{0}^{T}\left|f_{2, n_{k}}^{*}(t)\right|_{H}^{2} d t \\
\leq & J\left(f^{*}\right)-\frac{1}{2} \int_{0}^{T}\left|\left(u^{\circ}-u_{d}\right)(t)\right|_{H}^{2} d t-\frac{1}{2} \int_{0}^{T}\left|f^{\circ}(t)\right|_{H}^{2} d t \\
= & J\left(f^{*}\right)-J\left(f^{\circ}\right) .
\end{aligned}
$$

Thus, we have

$$
J\left(f^{\circ}\right)+\frac{1}{2} \limsup _{k \rightarrow \infty} \int_{0}^{T}\left|\left(f_{2, n_{k}}^{*}-f^{*}\right)(t)\right|_{H}^{2} d t \leq J\left(f^{*}\right) .
$$

Since $f^{*}$ is the optimal control to (OP), we see that

$$
\frac{1}{2} \limsup _{k \rightarrow \infty} \int_{0}^{T}\left|\left(f_{2, n_{k}}^{*}-f^{*}\right)(t)\right|_{H}^{2} d t=0 .
$$

Therefore, we observe from (5.9) and (5.11) that $f^{\circ}=f^{*}$ and

$$
f_{2, n_{k}}^{*} \longrightarrow f^{*} \quad \text { strongly in } L^{2}(0, T ; H) \quad \text { as } k \rightarrow \infty,
$$

hence, the convergence (3.2) holds.

Also, we infer from (5.10) and the uniqueness of solutions to $\left(\mathrm{CP} ; f^{*}, u_{0}\right)$ that $u^{\circ}=u^{*}$ and

$$
u_{n_{k}}^{*} \longrightarrow u^{*} \quad \text { strongly in } C([0, T] ; H) \quad \text { as } k \rightarrow \infty,
$$

hence, the convergence (3.3) holds.

Thus, the proof of Theorem 3.5 has been completed.

\section{Application}

In this section, we apply our abstract results (Theorems $3.2,3.3,3.4,3.5$ ) to a quasilinear parabolic PDE with a Signorini-Dirichlet-Neumann type mixed boundary condition as follows.

Problem (P). Find a function $u:[0, T] \longrightarrow L^{2}(\Omega)$ such that

$$
\begin{aligned}
& u_{t}-\nabla \cdot a(x, u, \nabla u)+g(u)=f(t, x) \quad \text { in }(0, T) \times \Omega \text {, } \\
& u \leq h(t), \quad \nu \cdot a(x, u, \nabla u) \leq 0 \\
& \text { and } \quad(u-h(t)) \nu \cdot a(x, u, \nabla u)=0 \quad \text { on }(0, T) \times \Gamma_{S} \text {, } \\
& u=h(t) \quad \text { on }(0, T) \times \Gamma_{D}, \\
& \nu \cdot a(x, u, \nabla u)=0 \quad \text { on }(0, T) \times \Gamma_{N}, \\
& u(0, \cdot)=u_{0} \quad \text { in } \Omega .
\end{aligned}
$$

Here, $\Omega$ is a bounded domain in $\mathbb{R}^{m}(m \geq 1)$ with smooth boundary $\Gamma:=\partial \Omega$, and $\Gamma$ admits a mutually disjoint decomposition such as

$$
\Gamma=\Gamma_{S} \cup \Gamma_{D} \cup \Gamma_{N},
$$


where $\Gamma_{S}, \Gamma_{D}$ and $\Gamma_{N}$ are measurable subsets of $\Gamma$. Also, $\nu$ is the outward normal vector on the boundary, the given function $g: \mathbb{R} \rightarrow \mathbb{R}$ is Lipschitz, and the function $h$ is given so that

$$
h \in W^{1,2}\left(0, T ; H^{1}(\Omega)\right) .
$$

Furthermore, the elliptic vector field $a(\cdot, \cdot, \cdot)$ is assumed to satisfy the following condition (A).

(A) $a(x, s, p)=\partial_{p} A(x, s, p)$ for some potential function $A(x, s, p)$. There exist constants $\mu>0, C_{5}>0$ and $C_{6}>0$ such that

$$
\begin{aligned}
{[a(x, s, p)-a(x, s, \hat{p})] \cdot(p-\hat{p}) } & \geq \mu|p-\hat{p}|^{2} \\
|a(x, s, p)|^{2}+|A(x, s, p)|+\left|\partial_{s} A(x, s, p)\right|^{2} & \leq C_{5}\left(1+|s|^{2}+|p|^{2}\right), \\
|a(x, s, p)-a(x, \hat{s}, p)| & \leq C_{6}(1+|p|)|s-\hat{s}|
\end{aligned}
$$

for all $x \in \Omega, s, \hat{s} \in \mathbb{R}, p, \hat{p} \in \mathbb{R}^{m}$.

Moreover, $a(\cdot, \cdot, \cdot)$ and $A(\cdot, \cdot, \cdot)$ satisfy the Carathéodory condition.

Now, we give the weak formulation of $(\mathrm{P})$. To do so, for each $t \in[0, T]$, we define the convex set $K(t)$ by

$$
K(t):=\left\{z \in H^{1}(\Omega) ; z \leq h(t) \text { on } \Gamma_{S} \text { and } z=h(t) \text { on } \Gamma_{D}\right\} .
$$

Here, we give the definition of solutions to $(\mathrm{P})$.

Definition 6.1. Given $f \in L^{2}\left(0, T ; L^{2}(\Omega)\right)$ and $u_{0} \in L^{2}(\Omega)$, a function $u:[0, T] \rightarrow H^{1}(\Omega)$ is called a solution of $(\mathrm{P})$, if the following items $(\mathrm{a})-(\mathrm{d})$ are satisfied.

(a) $u \in W^{1,2}\left(0, T ; L^{2}(\Omega)\right) \cap L^{\infty}\left(0, T ; H^{1}(\Omega)\right)$.

(b) $u \in K(t)$ for a.e. $t \in(0, T)$.

(c) For a.e. $t \in(0, T)$, the following inequality holds:

$$
\left(u_{t}, u-v\right)+\int_{\Omega} a(x, u, \nabla u) \cdot \nabla(u-v) d x+(g(u)-f, u-v) \leq 0
$$

for all $v \in K(t)$, where $(\cdot, \cdot)$ is the standard inner product on $L^{2}(\Omega)$.

(d) $u(0)=u_{0}$ in $L^{2}(\Omega)$.

Now, let us reformulate the problem $(\mathrm{P})$ as the abstract evolution equation $\left(\mathrm{CP} ; f, u_{0}\right)$. To do so, we define the time-dependent functional $\varphi^{t}(\cdot ; \cdot): L^{2}(\Omega) \times L^{2}(\Omega) \rightarrow \mathbb{R} \cup\{\infty\}$ by

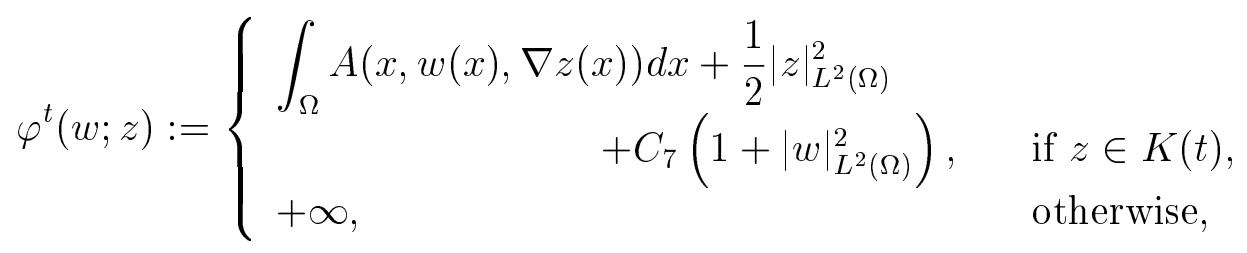


where $|\cdot|_{L^{2}(\Omega)}$ is the standard norm of $L^{2}(\Omega)$, and $C_{7}>0$ is the constant so that

$$
\begin{aligned}
& \varphi^{t}(w ; z) \geq \frac{1}{2}|z|_{L^{2}(\Omega)}^{2}+\frac{\mu}{4}|\nabla z|_{L^{2}(\Omega)}^{2}+|w|_{L^{2}(\Omega)}^{2}, \\
& \forall t \in[0, T], \forall z \in K(t), \forall w \in L^{2}(\Omega) .
\end{aligned}
$$

By the similar calculation in [17, Lemma 3.1], we can find the positive constant $C_{7}$ in (6.1). For the detailed calculation, see [17, Lemma 3.1].

Also, we define the single-valued operator $G(t, \cdot): L^{2}(\Omega) \rightarrow L^{2}(\Omega)$ and the function $\gamma: L^{2}(\Omega) \rightarrow \mathbb{R}$ by

$$
G(t, z):=g(z(\cdot))-z(\cdot) \text { in } L^{2}(\Omega), \quad \forall t \in[0, T]
$$

and

$$
\gamma(z):=\int_{\Omega} z^{+}(x) d x \text { for } z \in L^{2}(\Omega)
$$

respectively, where $z^{+}:=\max \{z, 0\}$.

Lemma 6.2. For each $t \in[0, T]$ and $r>0$, we put

$$
\alpha_{r}(t):=C_{8} \int_{0}^{t}\left|h^{\prime}(\tau)\right|_{H^{1}(\Omega)} d \tau \quad \text { for some (sufficient large) constant } C_{8}>0
$$

where $|\cdot|_{H^{1}(\Omega)}$ is the standard norm of $H^{1}(\Omega)$. Then, we have $\left\{\varphi^{t}\right\} \in \Phi_{\gamma}\left(\left\{\alpha_{r}\right\}\right)$ and $\{G(t, \cdot)\} \in \mathcal{G}_{\gamma}\left(\left\{\varphi^{t}\right\}\right)$.

Proof. We can show ( $\Phi 5)$ by taking

$$
\tilde{z}:=z-h(s)+h(t)
$$

for given $z \in K(s)$. Then, by the similar calculation in [17, Section 5.1], we can show $(\Phi 5)$.

Also, we can easily observe from (6.1)-(6.3) that $\left\{\varphi^{t}\right\} \in \Phi_{\gamma}\left(\left\{\alpha_{r}\right\}\right)$ and $G(t, \cdot) \in$ $\mathcal{G}_{\gamma}\left(\left\{\varphi^{t}\right\}\right)$. We omit the detailed proof. For the detailed arguments, we refer to $[17$, Sections 3.1 and 5.1], for instance.

As a direct application of Propositions 2.5 and 2.10, we get the following result of existence-uniqueness of solutions to $(\mathrm{P})$.

Proposition 6.3. Assume (A). Then, for each $f \in L^{2}\left(0, T ; L^{2}(\Omega)\right)$ and $u_{0} \in K(0)$, the problem $(\mathrm{P})$ has a unique solution $u$ on $[0, T]$.

Proof. By [17, Lemma 3.2], we easily see that the problem (P) is reformulated as in the following abstract form $\left(\mathrm{CP} ; f, u_{0}\right)$ :

$$
\left(\mathrm{CP} ; f, u_{0}\right)\left\{\begin{array}{l}
u^{\prime}(t)+\partial \varphi^{t}(u(t) ; u(t))+G(t, u(t)) \ni f(t) \quad \text { in } L^{2}(\Omega), \text { a.e. } t \in(0, T), \\
u(0)=u_{0} .
\end{array}\right.
$$


Taking account of Lemma 6.2, we can apply Propositions 2.5 and 2.10. Thus, we observe that the problem $(\mathrm{P})$ has a unique solution $u$ on $[0, T]$.

Furthermore, by applying Theorem 3.3, we see that the optimal control problem for (P) has at least one optimal control $f^{*} \in L^{2}\left(0, T ; L^{2}(\Omega)\right)$ :

$$
J\left(f^{*}\right)=\inf _{f \in L^{2}\left(0, T ; L^{2}(\Omega)\right)} J(f),
$$

where $J(\cdot)$ is the cost functional defined in (1.4) with $H:=L^{2}(\Omega)$.

Next, for each $n \in \mathbb{N}$, we consider the following approximating problem of $(\mathrm{P})$, denoted by $(\mathrm{P})_{n}$ :

Problem $(\mathbf{P})_{n}$. Find a function $u:[0, T] \longrightarrow L^{2}(\Omega)$ such that

$$
\begin{array}{cl}
\left(u_{n}\right)_{t}-\nabla \cdot a\left(x, u_{n}, \nabla u_{n}\right)+g\left(u_{n}\right)=f(t, x) & \text { in }(0, T) \times \Omega, \\
\nu \cdot a\left(x, u_{n}, \nabla u_{n}\right)=-\frac{u_{n}-h(t)}{\lambda_{n}} \cdot \chi_{D}-\frac{\left[u_{n}-h(t)\right]^{+}}{\lambda_{n}} \cdot \chi_{S} & \text { on }(0, T) \times \Gamma, \\
u_{n}(0, \cdot)=u_{0} & \text { in } \Omega,
\end{array}
$$

where $u_{0} \in K(0), \lambda_{n}:=\frac{1}{n}$ and $\chi_{D}\left(\right.$ resp. $\left.\chi_{S}\right)$ is the characteristic function of the set $(0, T) \times \Gamma_{D}\left(\operatorname{resp} .(0, T) \times \Gamma_{S}\right)$.

By the similar argument as the problem $(\mathrm{P})$, we can show the existence-uniqueness of solution to $(\mathrm{P})_{n}$ for each $n \in \mathbb{N}$. In fact, for any $t \in[0, T]$, we define the time-dependent of functional $\varphi_{n}^{t}(\cdot ; \cdot): L^{2}(\Omega) \times L^{2}(\Omega) \rightarrow \mathbb{R} \cup\{\infty\}$ by

$$
\varphi_{n}^{t}(w ; z):=\left\{\begin{array}{rr}
\int_{\Omega} A(x, w(x), \nabla z(x)) d x+\frac{1}{2}|z|_{L^{2}(\Omega)}^{2}+C_{7}\left(1+|w|_{L^{2}(\Omega)}^{2}\right) \\
\quad+\frac{1}{2 \lambda_{n}} \int_{\Gamma_{D}}|z-h(t)|^{2} d \Gamma+\frac{1}{2 \lambda_{n}} \int_{\Gamma_{S}}\left([z-h(t)]^{+}\right)^{2} d \Gamma, \\
+\infty, & \text { if } z \in H^{1}(\Omega), \\
\text { otherwise },
\end{array}\right.
$$

where $C_{7}>0$ is the same constant in $(6.1)$.

Then, we easily see that the problem $(\mathrm{P})_{n}$ is reformulated as in the abstract form $\left(\mathrm{CP} ; f, u_{0}\right)_{n}$ :

$$
\left(\mathrm{CP} ; f, u_{0}\right)_{n}\left\{\begin{array}{lr}
u_{n}^{\prime}(t)+\partial \varphi_{n}^{t}\left(u_{n}(t) ; u_{n}(t)\right)+G\left(t, u_{n}(t)\right) \ni f(t) & \text { in } L^{2}(\Omega), \\
u_{n}(0)=u_{0} . & \text { a.e. } t \in(0, T),
\end{array}\right.
$$

Clearly, for the function $\alpha_{r}$ defined in Lemma 6.2, we have $\left\{\varphi_{n}^{t}\right\} \in \Phi_{\gamma}\left(\left\{\alpha_{r}\right\}\right)$ and $\{G(t, \cdot)\} \in$ $\mathcal{G}_{\gamma}\left(\left\{\varphi_{n}^{t}\right\}\right)$. Therefore, by applying Propositions 2.5 and 2.10 , we see that for each $n \in \mathbb{N}$, $f \in L^{2}\left(0, T ; L^{2}(\Omega)\right)$ and $u_{0} \in K(0)$, the approximating problem $(\mathrm{P})_{n}$ has a unique solution $u_{n}$ on $[0, T]$.

Also, it is very easy to check the convergence assumption (K1) in Theorem 3.2, since we employ the standard approximation of the boundary by the penalty method in the 
problem $(\mathrm{P})_{n}$. Thus, by applying Theorem 3.2, we observe that the unique solution $u_{n}$ of $(\mathrm{P})_{n}$ converges to one $u$ of $(\mathrm{P})$ in the sense that

$$
u_{n} \rightarrow u \text { in } C\left([0, T] ; L^{2}(\Omega)\right), \quad\left(u_{n}\right)_{t} \rightarrow u_{t} \text { weakly in } L^{2}\left(0, T ; L^{2}(\Omega)\right) \quad \text { as } n \rightarrow \infty .
$$

Furthermore, by applying Theorems 3.4 and 3.5, we get the existence of an optimal control for $(\mathrm{P})_{n}$, and the relationship between the optimal control problems for $(\mathrm{P})$ and $(\mathrm{P})_{n}$.

\section{Acknowledgements}

The author wishes to thank Professor Karl-Heinz Hoffmann, Professor Nobuyuki Kenmochi and Professor Masahiro Kubo for their valuable comments and discussions.

\section{References}

[1] G. Akagi, Convergence of functionals and its applications to parabolic equations, Abstr. Appl. Anal., 2004, 907-933.

[2] H. Attouch, Variational Convergence for Functions and Operators, Pitman Advanced Publishing Program, Boston-London-Melbourne, 1984.

[3] H. Attouch and A. Damlamian, Problèmes d'évolution dans les Hilberts et applications, J. Math. Pures Appl., 54(1975), 53-74.

[4] V. Barbu, Optimal control of variational inequalities, Research Notes in Mathematics, 100, Pitman, London, 1984.

[5] Ph. Bénilan, Equations d'évolution dans un espace de Banach quelconque et application, Université de Paris-Sud, Publication Mathématique d'Orsay, 1972.

[6] H. Brézis, Opérateurs Maximaux Monotones et Semi-Groupes de Contractions dans les Espaces de Hilbert, North-Holland, Amsterdam-London-New York, 1973.

[7] A. Damlamian, Non Linear Evalution Equations with Variable Norms, Thesis, Harvard University, 1974.

[8] A. Friedman, Optimal control for parabolic variational inequalities, SIAM J. Control Optim., 25(1987), 482-497.

[9] N. Fujiyama and J. Watanabe, Convergence of subdifferentials and the penalty method, Rep. Univ. Electro-Comm., 28(1977/78), 245-258.

[10] S. Hu and N. S. Papageorgiou, Time-dependent subdifferential evolution inclusions and optimal control, Mem. Amer. Math. Soc., 133(1998), no. 632, viii+81 pp.

[11] R. Kano, Y. Murase and N. Kenmochi, Nonlinear evolution equations generated by subdifferentials with nonlocal constrains, Submitted to the Banach Center Publications, Institute of Mathematics Polish Academy of Sciences. 
[12] N. Kenmochi, Some nonlinear parabolic variational inequalities, Israel J. Math., 22(1975), 304-331.

[13] N. Kenmochi, Solvability of nonlinear evolution equations with time-dependent constraints and applications, Bull. Fac. Education, Chiba Univ., 30(1981), 1-87.

[14] N. Kenmochi and N. Yamazaki, Global attractors for multivalued flows associated with subdifferentials, pp. 135-144, in Elliptic and Parabolic Problems, (Rolduc/Gaeta, 2001), World Sci. Publ., River Edge, NJ, 2002.

[15] M. Kubo, Characterization of a class of evolution operators generated by timedependent subdifferentials, Funk. Ekvac., 32(1989), 301-321.

[16] M. Kubo and N. Yamazaki, Quasilinear parabolic variational inequalities with timedependent constraints, Adv. Math. Sci. Appl., 15(2005), 335-354.

[17] M. Kubo and N. Yamazaki, Elliptic-parabolic variational inequalities with timedependent constraints, Discrete Contin. Dyn. Syst., 19(2007), 335-359.

[18] J.-L. Lions, Optimal control of systems governed by partial differential equations, Die Grundlehren der mathematischen Wissenschaften, Band 170, Springer-Verlag, New York-Berlin, 1971.

[19] F. Mignot and J.-P. Puel, Optimal control in some variational inequalities, SIAM J. Control Optim., 22(1984), 466-476.

[20] U. Mosco, Convergence of convex sets and of solutions of variational inequalities, Advances in Math., 3(1969), 510-585.

[21] M. Ôtani, Nonmonotone perturbations for nonlinear parabolic equations associated with subdifferential operators, Cauchy problems, J. Differential Equations, 46(1982), 268-299.

[22] M. Ôtani, Nonmonotone perturbations for nonlinear parabolic equations associated with subdifferential operators, periodic problems, J. Differential Equations, 54(1984), 248-273.

[23] M. Ôtani, Nonlinear evolution equations with time-dependent constraints, Adv. Math. Sci. Appl., 3(1993/94), Special Issue, 383-399.

[24] S.-U. Ryu, Optimal control problems governed by some semilinear parabolic equations, Nonlinear Anal., 56(2004), 241-252.

[25] K. Shirakawa, A. Ito, N. Yamazaki and N. Kenmochi, Asymptotic stability for evolution equations governed by subdifferentials, pp. 287-310, in Recent developments in domain decomposition methods and flow problems, GAKUTO Internat. Ser. Math. Sci. Appl., 11, Gakkōtosho, Tokyo, 1998.

[26] U. Stefanelli, Nonlocal quasivariational evolution problems, J. Differential Equations, 229(2006), 204-228. 
[27] Y. Yamada, On evolution equations generated by subdifferential operators, J. Fac. Sci. Univ. Tokyo Sect. IA Math., 23(1976), 491-515.

[28] N. Yamazaki, Attractors of asymptotically periodic multivalued dynamical systems governed by time-dependent subdifferentials, Electron. J. Differential Equations, 2004, No. 107, pp. 1-22.

[29] N. Yamazaki, A class of nonlinear evolution equations governed by time-dependent operators of subdifferential type, pp. 280-296, In Mathematical Approach to Nonlinear Phenomena; Modelling, Analysis and Simulation, GAKUTO Intern. Ser. Math. Appl., 23, Gakkotosho, Tokyo, 2005.

[30] S. Yotsutani, Evolution equations associated with the subdifferentials, J. Math. Soc. Japan, 31(1979), 623-646. 